GENERIC FINITENESS OF EQUILIBRIUM OUTCOME DISTRIBUTIONS FOR SENDER-RECEIVER

CHEAP-TALK GAMES

by

In-Uck Park

Discussion Paper No. 269, September 1993

\author{
Center for Economic Research \\ Department of Economics \\ University of Minnesota \\ Minneapolis, MN 55455
}




\title{
Generic Finiteness of Equilibrium Outcome Distributions for Sender-Receiver Cheap-Talk Games
}

\author{
In-Uck Park* \\ Department of Economics \\ University of Minnesota \\ 271 19th Avenue S. \\ Minneapolis, MN 55455 \\ U.S.A. \\ and \\ Department of Economics \\ University of Bristol \\ 8 Woodland Road \\ Bristol, BS8 1TN \\ U.K.
}

September, 1993

\begin{abstract}
This paper establishes the generic finiteness of equilibrium outcome distributions for Sender-Receiver cheap-talk games. An equilibrium in a Sender-Receiver cheap-talk game is said to be in reduced form if every message is used by at least one type and no two messages provoke the same response. It is shown that, for a generic set of utilities on outcomes, a Sender-Receiver cheap-talk game has a finite number of reduced form equilibria. A corollary is that, for generic utilities, the set of probability distributions over outcomes generated by equilibria is finite. Because of the identification of terminal nodes for utility purposes, Sard's theorem is not applicable in the way it was used in Kreps and Wilson (1982), and a structurally different proof strategy is developed. Some additional characterization of the equilibria are obtained in the process of the proof.

Keywords: cheap-talk games, reduced form equilibrium, outcome distribution, configuration, induced utility plane, $\chi$-value

$J E L$ classification code: $\mathrm{C} 72$

Correspondence: University of Bristol address above

* I acknowledge my special thanks to Andy McLennan for the continuing encouragement and invaluable guidance in every respect. I also would like to thank Ed Green for very helpful advice and comments. I am responsible for remaining errors.
\end{abstract}




\section{Introduction}

This paper establishes the generic finiteness of equilibrium outcome distributions (these terms are clarified below) in a simple class of "pure communication games," namely, games in which a privately informed "Sender" sends a utility-irrelevant message concerning her type to a "Receiver" who, upon receiving the message, takes an action. In this paper, messages do not affect utility and play only an informational signaling role. We define "outcomes" to be type-action pairs; the space of outcomes is the domain of agents' utility functions. Given an extensive form game derived in the obvious way from a particular message space, multiple terminal nodes correspond to the same outcome if they differ only in their message components. Often called Sender-Receiver cheap-talk games, this class of games is introduced by Crawford and Sobel (1982) and is different from the signaling games studied in Cho and Kreps $(1987)^{(1)}$ in one respect: messages do not affect utility.

In this paper, a subset of a finite dimensional linear space is said to be "generic" if the complement is a finite union of semi-algebraic sets ${ }^{(2)}$ whose closure has measure zero. An equilibrium in a Sender-Receiver cheap-talk game is said to be in reduced form if every message is used by at least one type and no two messages provoke the same response. It is shown that, for a generic subset of the space of utilities on outcomes, a Sender-Receiver cheap-talk game has a finite number of reduced form equilibria. Since every equilibrium has a reduced form reduction with the same outcome distribution, the conceptual essence of the paper follows as a simple corollary, namely, a Sender-Receiver cheap-talk game has a finite number of outcome distributions generated by equilibria.

Equilibrium theories explain the relationship between exogenous variables (given environment) and endogenous variables (determined by the theory) as solutions to a system of equations. In addition to the issue of the existence of a solution, one is concerned about the determinacy of the solution that a theory prescribes. In particular, if there are infinitely many solutions in a small neighborhood of a point, the theory's explanation for the given situation is indeterminate at least near that point. Ideally, a theory provides a completely

(1) As they indicate in their paper, signaling games have been studied in various contexts prior to their paper.

(2) Roughly, a set is semi-algebraic if it can be obtained by finite unions and/or intersections of solution sets to polynomial (in)equalities. See subsection 3.2 for a definition of semi-algebraic sets. 
determinate explanation if the endogenous variables are expressed as a continuous function of exogenous variables. But, both general equilibrium theory and game theory do not generally determine the solution in this way. However, if the hypotheses of the implicit function theorem are satisfied by the equilibrium conditions at each solution point, the ideal situation is realized locally: the solution is locally unique and is persistent under a small perturbation of exogenous variables. Therefore, the comparative statics provides unambiguous predictions of the theory on the effects of small variations in exogenous variables, and the implication of the theory is not invalidated by the possibility of a small error in exogenous variables.

In game theory, typically the determinacy of equilibria is investigated taking utilities as given variables, which is the point of view taken in this paper. In a finite game, the local uniqueness of equilibrium is equivalent to finiteness of equilibria because the strategy space is compact. The most general result of finite equilibria is not obtainable because a continuum of equilibria always arises for special utilities (e.g, the same utility for every outcome). Hence, as in general equilibrium theory, a "generic" property is sought for.

In extensive form games, the behavior at unreached nodes may vary without affecting the equilibrium conditions, giving rise to a continuum of equilibria. These variations are ineffective in the sense that the variation part has no role in determining the terminal nodes reached by equilibria. Hence, for extensive form games, the determinacy of equilibrium probability distributions on terminal nodes is established by Kreps and Wilson (1982). In pure communication games, the strategic meanings of messages are determined within equilibria according to how they are used, and because many messages may be used as "synonyms," equilibria are inherently indeterminate. This kind of indeterminacy is not present in previous studies on the determinacy of games in which every move has a unique role. In this respect, the issue of determinacy in pure communication games has a special significance pertaining to the utility-irrelevant and unverifiable nature of language.

Generic finiteness of equilibria was first established by Debreu (1970) for pure exchange economies and the investigation followed for varied game forms. Harsanyi (1973) shows that a finite normal form game has an odd (hence, finite) number of equilibria for 
generic utilities on the set of pure strategy profiles. ${ }^{(3)}$ Kreps and Wilson (1982) resolves the issue for extensive form games; given an extensive form game, the set of probability distributions on the terminal nodes generated by Nash equilibria is finite for generic utilities on the set of terminal nodes.

However, games may have sets of terminal nodes that are naturally regarded as $a$ priori equivalent due to the nature of the phenomenon being modelled. For these games, the "relevant" utility space is a linear subspace of the utility space on all terminal nodes, and the Kreps and Wilson's result is not applicable. In this regard, McLennan (1991) investigates more general game forms in which terminal nodes are mapped to an outcome space to which utility is assigned. Although he establishes positive results for some special cases, McLennan presents an example that refutes the finiteness of equilibrium outcome distributions for generic utilities when terminal nodes are mapped to mixed outcomes (probability distributions over pure outcomes). The case in which terminal nodes are mapped to pure outcomes is still an open question. Games involving pure communication (utility-irrelevant messages) are an interesting subclass of this case. This paper resolves the question for Sender-Receiver cheap-talk games which are simple forms of this subclass, with the hope of providing an insight to general pure communication games.

In a Sender-Receiver cheap-talk game, the outcome space is the product of the set of Sender types and the set of actions available to the Receiver. Hence, terminal nodes reached from the same type and same action (but different messages) are identified as the same outcome so that, for nontrivial message spaces, the dimension of the utility space of an agent is smaller than the number of terminal nodes. It turns out, therefore, Sard's theorem is not applicable here in the way it was used to prove generic finiteness in Kreps and Wilson (1982). A structurally different proof strategy is developed in this paper.

To facilitate the discussion of methodology, a description of the game is in order. Denote the probability space on a finite set $S$ by

$$
\Delta(S) \equiv\left\{p \in \mathbb{R}_{+}^{S}: \sum_{s \in S} p_{s}=1\right\}
$$

(3) Harsanyi proves a stronger result that finite normal form games are "regular" for generic utilities. For the definition of regular game, refer to his paper. 
and let $\Delta^{\circ}(S)$ be the relative interior of $\Delta(S)$. A Sender-Receiver cheap-talk game is represented by $\Gamma=(\mathbf{A}, \mathbf{T}, \pi, u, v)$ where $\mathbf{A}$ is a finite set of actions available for the Receiver, $\mathbf{T}$ is a finite set of the Sender types, $\pi \in \Delta^{\circ}(\mathbf{T})$ is the prior belief of the Receiver on the distribution of Sender types and $u \in \mathbb{R}^{\mathbf{A} \times \mathbf{T}}$ and $v \in \mathbb{R}^{\mathbf{A} \times \mathbf{T}}$ are the utilities of the Sender and the Receiver, respectively. ${ }^{(4)}$ The characteristics $(\mathbf{A}, \mathbf{T}, \pi)$ is called a game form.

Let $\mathbf{M}$ be a finite message space. ${ }^{(5)}$ A talking strategy of the Sender is a function $\tau: \mathbf{T} \rightarrow \Delta(\mathbf{M})$, and an action strategy of the Receiver is a function $\alpha: \mathbf{M} \rightarrow \Delta(\mathbf{A})$. A Bayesian Nash equilibrium is a pair of strategies $(\tau, \alpha)$ that satisfies $i)$ given $\alpha$, each type sends only those messages that induce the highest expected utility for that type, and $i i$ ) for each message, $\alpha$ prescribes a best response of the Receiver based on the Bayesian updating of the beliefs under $\tau$. Throughout the paper, an equilibrium refers to a Bayesian Nash equilibrium. In fact, the properties that we exploit to establish the main result are weaker than the full implication of a Bayesian Nash equilibrium; the "best response" property of the Sender is not exploited. Hence, the result we get is somewhat stronger in the sense that it applies to a weaker concept than a Bayesian Nash equilibrium.

In a game $\Gamma$, the set of outcomes is defined to be $\mathbf{A} \times \mathbf{T}$. The outcome distribution generated by $(\tau, \alpha)$ is the probability that each outcome is reached when the game is played according to $(\tau, \alpha)$. The subject of this paper is the cardinality of outcome distributions generated by equilibria of the game for generic utilities $(u, v)$.

The function of messages is to induce certain responses from the Receiver by affecting the belief (of the Receiver). In this sense, messages are strategically equivalent if they induce the same response. Also, unused messages play no role in determining outcome distribution. A reduced form equilibrium (as defined earlier) is without these kinds of redundancy of messages. As is discussed in section 2, an equilibrium that is not in reduced form can be transformed to a reduced form equilibrium with the same outcome distribution by eliminating unused messages and identifying messages provoking the same response. Hence, we restrict our attention to reduced form equilibria. The main theorem of this paper

(4) An alternative representation of a utility of either agent is a linear function from $\mathbb{R}^{\mathbf{A}}$ to $\mathbb{R}^{\mathbf{T}}$.

(5) Every result of this paper is easily seen to hold for a countably infinite message space also. 
states that for generic utilities there are only a finite number of reduced form equilibria. It is an immediate corollary of this theorem that, for generic utilities, there are a finite number of outcome distributions generated by Bayesian Nash equilibria.

Consider an equilibrium $(\tau, \alpha)$ of a game $\Gamma$. For each message used according to $\tau$, the bisupport of the message is a pair consisting of the set of types that use that message with a positive probability ("type component"), and the set of actions that are used with a positive probability in response to that message ("action component"). The configuration determined by an equilibrium is the collection of bisupports of all used messages. We say an equilibrium is in the configuration that it determines and an outcome distribution is consistent with a configuration if it is generated by an equilibrium in that configuration. We will investigate all possible configurations and outcome distributions that are consistent with these configurations.

Next, let us consider a certain configuration. The expected utility vectors of the Sender induced by equilibria in that configuration, are shown to be contained in a certain affine subspace ${ }^{(6)}$ determined by the configuration and the utility of the Sender. The generic dimension of this affine subspace is the main task of section 4 . The results are obtained for all configurations necessary for our purpose; results are established for a special class of configurations first and are extended to more general classes. In section 5, configurations with positive values of the above mentioned generic dimension are investigated of their compatibility with generic utilities of the Receiver from the viewpoint of Green and Osband (1991), and the main theorem is established.

This rather long proof produces some additional implications for Sender-Receiver cheap-talk games.

It is shown that, for generic utilities, every reduced form equilibrium uses at most as many messages as the number of Sender types. This observation works as a rationale for the assumption of a finite message space widely adopted in communication literature. In fact, the message space may be reduced down to the number of Sender types for the purpose of finding reduced form equilibria for generic utilities. Moreover, generically, every configuration has at most one reduced form equilibrium in it, and a relatively small

(6) See subsection 2.2 for a definition of affine subspace. 
number of configurations consisting of \#T or less bisupports may have reduced form equilibria in them. This information may be useful in the exercise of computing equilibria. Third implication is that, for each game form, there is an upper bound for the number of equilibrium outcome distributions that applies for generic utilities.

The essential feature of Sender-Receiver cheap-talk games is the coordination between two players on the information that the language transmits. The question of how the coordination is finally reached is an important issue which this paper does not address. However, the coordination may be expected to be easier to reach if there are only a finite number of "rational" ways of using the language, particularly near an equilibrium point. This remark, however, is to be understood as a naive observation due to the lack of coordination process specification on the one hand and the highly complex nature of human language on the other.

Section 2 presents a formal framework and a comprehensive outline of the analysis. Section 3 illustrates a simple 2-type case to give a basic idea of the more complex general analysis that ensues. A discussion of semi-algebraic sets and affine subspaces is also in section 3.

\section{Main Results and Analytic Framework}

\subsection{Reduced form equilibria and main results}

In this subsection, Bayesian Nash equilibria and reduced form equilibria are formally defined and the main results of this paper is stated.

Given a set of Sender types $\mathbf{T}$ and a set of actions $\mathbf{A}$, the following notation will be used: $u \in \mathbb{R}^{\mathrm{A} \times \mathrm{T}}$ is a tuple $\left(u^{a}\right)_{a \in \mathrm{A}}$ where $u^{a} \in \mathbb{R}^{\mathrm{T}}$ is the utility vector of all possible types of Sender induced by action $a$. Alternatively, $u$ can be expressed as a tuple $\left(u_{t}\right)_{t \in \mathbf{T}}$ where $u_{t} \in \mathbb{R}^{\mathbf{A}}$ is the utility vector of type $t$ Sender induced by all available actions in $\mathbf{A}$. Analogous notation is adopted for $v$, a utility of the Receiver. A talking strategy $\tau$ is a tuple $\left(\tau_{t}\right)_{t \in \mathbf{T}}$ where $\tau_{t}$ is the strategy of type $t$ Sender. For $p \in \Delta(\mathbf{T}), p_{t}$ is the probability of type $t$ according to $p$.

Given a game $(\mathbf{A}, \mathbf{T}, \pi, u, v)$, consider a talking strategy $\tau: \mathbf{T} \rightarrow \Delta(\mathbf{M})$ where $\mathbf{M}$ is a finite message space. A message $m$ is a used message if $\sum_{t \in \mathbf{T}} \tau_{t}(m)>0$ and is an 
unused message if otherwise. For a used message $m$, the posterior belief of $m$ induced by $\tau$, denoted by $h_{\tau}(m) \in \Delta(\mathbf{T})$, is defined as;

$$
\left(h_{\tau}(m)\right)_{t} \equiv \frac{\pi_{t} \tau_{t}(m)}{\sum_{t^{\prime} \in \mathbf{T}} \pi_{t^{\prime}} \tau_{t^{\prime}}(m)} \quad \text { for all } t \in \mathbf{T}
$$

Let $B R(p ; v)$ be the set of pure best responses of the Receiver when the Receiver believes that $p \in \Delta(\mathbf{T})$ is the distribution of the type of the Sender:

$$
B R(p ; v) \equiv\left\{a \in \mathbf{A}: p \cdot v^{a} \geq p \cdot v^{a^{\prime}}, \forall a^{\prime} \in \mathbf{A}\right\}
$$

A Bayesian Nash equilibrium of a game $(\mathbf{A}, \mathbf{T}, \pi, u, v)$ is a pair of strategies $(\tau, \alpha)$ on a message space $\mathbf{M}$ that satisfies $^{(7)}$;

i) for all $t \in \mathbf{T},\left[\tau_{t}(m)>0\right] \Rightarrow\left[\alpha(m) \cdot u_{t} \geq \alpha\left(m^{\prime}\right) \cdot u_{t}, \forall m^{\prime} \in \mathbf{M}\right]$, and

ii) $\left[\operatorname{supp}(\alpha(m)) \in B R\left(h_{\tau}(m) ; v\right)\right]$ if $m$ is used, and $[\operatorname{supp}(\alpha(m)) \in B R(p ; v)$ for some $p \in \Delta(\mathbf{T})]$ if $m$ is unused.

where supp represents the support. In this paper, an equilibrium refers to a Bayesian Nash equilibrium.

As discussed earlier, unused messages and multiple messages that provoke the same response give rise to redundancy of messages. Recall that an equilibrium $(\tau, \alpha)$ is in reduced form if every message is used and $\alpha(m) \neq \alpha\left(m^{\prime}\right)$ for any two distinct messages $m$ and $m^{\prime}$. As the next lemma implies, we only need to consider reduced form equilibria to show the generic finiteness of equilibrium outcome distributions.

Lemma 2.1: For any equilibrium, there is a reduced form equilibrium with the same outcome distribution.

Proof: Let $(\tau, \alpha)$ be an equilibrium with a message space $\mathbf{M}$ which is not in reduced form. Define an equivalence relation on $\mathbf{M}$ as follows; $m$ and $m^{\prime}$ are equivalent if $\alpha(m)=\alpha\left(m^{\prime}\right)$. Let $[m]$ be the equivalence class of $m$. Define an induced message space $\tilde{\mathbf{M}}$ to be the set

(7) This definition is equivalent to the sequential equilibria for Sender-Receiver cheap-talk games; the additional requirement of the sequential equilibrium is the "consistency" of the belief (off equilibrium). Since every information set is directly reachable from every initial node, any belief specification at unreached nodes is implementable with "negligible" changes in the beliefs of reached nodes. 
of equivalence classes of all used messages in $M$. This is a situation where messages that provoke the same response are identified as a single message. The original behavior of the agents is still in equilibrium after the identification; the Sender's optimality is immediate and the Receiver's optimality is preserved by the "sure thing principle." By construction, it is in reduced form and the outcome distribution is not affected by the identification.

Given a game form $(\mathbf{A}, \mathbf{T}, \pi)$, the space of utilities of the agents of the game is commonly $\mathbb{R}^{\mathbf{A} \times \mathbf{T}}$. To distinguish between the Sender and the Receiver, let $\mathcal{U}$ denote the utility space of the Sender and $\mathcal{V}$ denote that of the Receiver. The goal of this paper is to show that for generic $(u, v)$ in the utility space $\mathcal{U} \times \mathcal{V}$, the game $\Gamma=(\mathbf{A}, \mathbf{T}, \pi, u, v)$ has a finite number of outcome distributions that are generated by Bayesian Nash equilibria.

This goal is accomplished by investigating the reduced form equilibria in every possible configuration. In fact, we establish a stronger generic characterization of the reduced form equilibria in relation to the configurations that they determine; according to certain criteria, each configuration either generically has no reduced form equilibrium in it or generically has at most one reduced form equilibrium in it. The precise characterizatrion and criteria are developed in later sections. In this subsection, the principal characterization of reduced form equilibria are stated in Theorem 2.2, from which the generic finiteness conclusion (Theorem 2.3 and Corollary 2.4 ) is deduced.

Theorem 2.2: Given a game form $(\mathbf{A}, \mathbf{T}, \pi)$, there is a generic subset $G$ of $\mathcal{U} \times \mathcal{V}$ such that, for all $(u, v) \in G$,

(a) every reduced form equilibrium uses at most as many messages as the number of Sender types, and

(b) for each configuration, there is at most one reduced form Bayesian Nash equilibrium in it.

Proof: Deferred to sections 4 and 5.

By part (a) of Theorem 2.2, for generic utilities, every reduced form equilibrium is in a configuration consisting of \# $\mathbf{T}$ or fewer bisupports. Since there are a finite number of distinct bisupports, part (b) establishes:

Theorem 2.3: Given a game form $(\mathbf{A}, \mathbf{T}, \pi)$, there is a generic subset $G$ of $\mathcal{U} \times \mathcal{V}$ such 
that, for all $(u, v) \in G$, the set of reduced form Bayesian Nash equilibria of $(\mathbf{A}, \mathbf{T}, \pi, u, v)$ is finite.

By virtue of Lemma 2.1, we have:

Corollary 2.4: Given a game form $(\mathbf{A}, \mathbf{T}, \pi)$, there is a generic subset $G$ of $\mathcal{U} \times \mathcal{V}$ such that, for all $(u, v) \in G$, the set of outcome distributions that are generated by Bayesian Nash equilibria of $(\mathbf{A}, \mathbf{T}, \pi, u, v)$ is finite:

\subsection{Analytic framework}

This subsection formalizes the analytic framework for the investigation of reduced form equilibria in each configuration, and explains the outline of the analysis that establishes Theorem 2.2.

A formulation of configurations is in order for further discussion. Prior to that, we make two observations on the configurations determined by equilibria. First, if a configuration is determined by an equilibrium, every type belongs to the type component of at least one bisupport because every type uses at least one message. This means that the union of type components of all bisupports in the configuration is the whole set of Sender types. Hence, we only need to investigate configurations with this property. Secondly, we show in next two Lemmas that, for generic utilities, no reduced form equilibrium determine a configuration with two identical bisupports. This observation simplifies notation for configurations in the subsequent discussion.

Lemma 2.5: Given a game form $(\mathbf{A}, \mathbf{T}, \pi)$, there is a generic subset $F$ of $\mathcal{V}$ such that, for all $v \in F, \#_{B R}(p ; v) \leq \#_{s u p p}(p)$ for all $p \in \Delta(\mathbf{T})$.

Proof: Because a finite intersection of generic sets is generic, it suffices to show that for generic $v, \# B R(p ; v) \leq \# \mathbf{T}$ for all $p \in \Delta(\mathbf{T})$. Let $k=\# \mathbf{T}$. To show that the proposed property is violated only in a negligible set, consider the set

$$
\left\{v \in \mathcal{V}: p \cdot v^{a_{1}}=p \cdot v^{a_{2}}=\cdots=p \cdot v^{a_{k+1}} \text { for some } p \in \Delta(\mathbf{T})\right\}
$$


where $a_{1}, \ldots, a^{k+1}$ are distinct actions. Notice this set satisfies

$$
\left(\begin{array}{c}
v^{a_{1}}-v^{a_{2}} \\
v^{a_{1}}-v^{a_{3}} \\
\vdots \\
v^{a_{1}}-v^{a_{k+1}}
\end{array}\right) \cdot p=0
$$

where $v^{a}$ is a row vector. Since $p \neq 0$, the above equality implies that the matrix on the left hand side is singular. Therefore, the above defined set is a negligible set. Since there are a finite number of collections of $k+1$ distinct actions, the proof is complete.

Lemma 2.6: Given a game form $(\mathbf{A}, \mathbf{T}, \pi)$, there is a generic subset $G$ of $\mathcal{U} \times \mathcal{V}$ such that, for all $(u, v) \in G$, no reduced form equilibrium of $(\mathbf{A}, \mathbf{T}, \pi, u, v)$ determines a configuration that has two identical bisupports.

Proof: Suppose an equilibrium determines a configuration with two identical bisupports consisting of $A \subset \mathbf{A}$ and $T \subset \mathbf{T}$ such that $\#_{A} \leq \# T$. (By Lemma 2.6, we only need to consider these bisupports.) Denote the two messages corresponding to this bisupport by $m$ and $m^{\prime}$. Let $u_{T}^{*} \in \mathbb{R}^{T}$ denote the equilibrium expected utility vector for Sender types in $T$. Since every type in $T$ uses both $m$ and $m^{\prime}$, the expected utility of Sender types in $T$ from the responses to $m$ and $m^{\prime}$ should be $u_{T}^{*}$. However, the set of all expected utility vectors for Sender types in $T$ that can be realized by responses mixed over $A$, is the convex hull of the utility vectors of all actions in $A$ for Sender types in $T$. (These utility vectors are elements of $\mathbb{R}^{T}$.) Since ${ }_{A} \leq \#_{T}$, these utility vectors are linearly independent for generic utilities. Therefore, for generic utilities, there is a unique response mixed over $A$ that induces the equilibrium expected utility vector for $T\left(u_{T}^{*}\right)$, which in turn means that the responses to $m$ and $m^{\prime}$ are the same. This contradicts to the equilibrium being in reduced form . Therefore, we conclude that, for generic utilities, there is no reduced form equilibrium in a configuration that has two copies of the bisupport $(A, T)$. Since there are finitely many distinct bisupports and a finite intersection of generic sets is generic, the proof is complete.

Due to Lemma 2.6, for generic utilities, every reduced form equilibrium determines a configuration consisting of distinct bisupports. In this respect, we only consider configurations with this property in the remainder of the paper, and a configuration always 
refers to a configuration with this property unless otherwise indicated. Accordingly, the following formulation of configurations comprises only these configurations.

Definition 2.7 : Given underlying sets of actions $\mathbf{A}$ and of Sender types T, a bisupport on $\{\mathbf{A}, \mathbf{T}\}$ is a pair $(A, T)$ where $A$ (called action component) is a nonempty subset of $\mathbf{A}$, and $T$ (called type component) is a nonempty subset of $\mathbf{T}$. $A$ configuration $\mathcal{C}$ on $\{\mathbf{A}, \mathbf{T}\}$ is a nonempty set of bisupports on $\{\mathbf{A}, \mathbf{T}\}$. When the underlying sets of actions and Sender types are obvious, we will omit specification of them. $A$ subconfiguration of $\mathcal{C}$ is a nonempty subset of $\mathcal{C}$. The cardinality of a configuration $\mathcal{C}, \#^{\mathcal{C}}$, is the number of bisupports in $\mathcal{C}$. $A$ configuration $\mathcal{C}$ on $\{\mathbf{A}, \mathbf{T}\}$ is just if $\#_{A} \leq \#_{T}$ for every bisupport $(A, T)$ in $\mathcal{C}$ and $U_{\mathcal{C}} T=\mathbf{T}$.

Remark 2.8 : In light of Lemma 2.5, we only need to consider configurations such that $\#_{A} \leq \#_{T}$ for every bisupport $(A, T)$. Moreover, since every type uses at least one message, every equilibrium determines a configuration such that the union of all type components is the set of all Sender types. Therefore, we only need to consider just configurations.

Given a game from $(\mathbf{A}, \mathbf{T}, \pi)$, our agenda is to show that, for generic utilities, only a finite number of configurations have reduced form equilibria in them and moreover, these configurations have only a finite number of reduced form equilibria in them. We focus on two necessary conditions that a configuration $\mathcal{C}$ should satisfy if, for a certain utility $(u, v)$, there is a reduced form equilibrium in it. Condition (SO) below is necessary for the optimality of the Sender and (RO) is necessary for that of the Receiver. By an expectation vector of the Sender, we mean a vector of expected utility levels of the Sender (one level for each type) from a certain action strategy of the Receiver. The space of expectation vectors of the Sender is $\mathbb{R}^{\mathrm{T}}{ }^{(8)}$

(SO) There is an expectation vector $u^{*} \in \mathbb{R}^{\mathrm{T}}$ (to be regarded as an equilibrium expected utility vector) of the Sender with the following property; for each bisupport $(A, T)$ in $\mathcal{C}$, there is a response $\alpha((A, T))$ mixed over $A$ such that $\alpha((A, T)) \cdot u_{t}=u_{t}^{*}$ for all $t \in T$.

(8) An expectation vector is the expected utility levels of Sender induced by an equilibrium in a given game. This needs to be distinguished from a utility (function) of Sender which is a parameter of the game. Recall that the space of the utilities of Sender is $\mathbb{I} R^{\mathbf{A}} \times \mathbf{T}$. 
(RO) There is a talking strategy $\tau: \mathbf{T} \rightarrow \Delta(\mathcal{C})$ such that, for all $(A, T) \in \mathcal{C}$, the following properties hold; i) $\tau_{t}((A, T))>0$ if and only if $t \in T$, and ii) $A \subset B R\left(h_{\tau}((A, T)) ; v\right)$. (Since each bisupport corresponds to a message used in an equilibrium, the configuration is identified as the message space.)

These conditions are weaker than the optimality conditions of the agents because the "best response" property of Sender is not incorporated. Hence, conclusions derived from these two conditions (as are the results of this paper) are actually stronger than the results stated in subsection 2.1. But, it may be of purely mathematical interest.

Our agenda is accomplished by classifying configurations into the following four categories (not necessarily exclusively) according to certain criteria, and showing that only a finite number of configurations belong to category (IV):

(I) For generic utilities of the Sender, if $u^{*}$ and $\{\alpha((A, T)):(A, T) \in \mathcal{C}\}$ satisfy (SO), then there are distinct bisupports $(A, T)$ and $\left(A^{\prime}, T^{\prime}\right)$ in $\mathcal{C}$ such that $\alpha((A, T))=$ $\alpha\left(\left(A^{\prime}, T^{\prime}\right)\right)$. (So that any equilibrium in $\mathcal{C}$ could not be in reduced form.)

(II) For generic utilities of the Sender, condition (SO) is violated with respect to $\mathcal{C}$.

(III) For generic utilities of the Receiver, condition (RO) is violated with respect to $\mathcal{C}$.

(IV) For generic utilities, there is at most one strategy pair $(\tau, \alpha)$ that satisfies (OS) and (OR) with respect to $\mathcal{C}$.

To develop criteria for classifying configurations into four categories above, we need some terminology and notation: Let $X$ be a subset of a finite dimensional linear space $\mathcal{L}$. An affine combination of points $x_{1}, \ldots, x_{n}$ in $X$ is $\gamma_{1} x_{1}+\cdots+\gamma_{n} x_{n}$ where $\gamma_{j} \in \mathbb{R}$ and $\sum_{j=1}^{n} \gamma_{j}=1$. The affine hull of $X$ is defined as

$$
H(X) \equiv\{z \in \mathcal{L}: z \text { is an affine combination of points in } X\}
$$

An affine subspace of $\mathcal{L}$ is a subset of $\mathcal{L}$ closed under affine combination. It is easy to see that an affine subspace is the affine hull of a finite subset (possibly empty) of $\mathcal{L}$, and vice versa. ${ }^{(9)}$ The affine hull of $X, H(X)$, needs to be distinguished from $\operatorname{span}(X)$ :

$$
\operatorname{span}(X) \equiv\{z \in \mathcal{L}: z \text { is a linear combination of points in } X\} .
$$

(9) Notice also that an affine hull is either empty or a linear subspace translated by a vector. 
Recall the space of expectation vectors of the Sender is $\mathbb{R}^{\mathbf{T}}$ where $\mathbf{T}$ is the set of Sender types. For a nonempty subset $T$ of $\mathbf{T}$, let $\mathbb{R}^{T}=\left\{x \in \mathbb{R}^{\mathbf{T}}: x_{t}=0\right.$ for all $\left.t \notin T\right\}$ and let $\phi_{T}: \mathbb{R}^{\mathbf{T}} \rightarrow \mathbb{R}^{T}$ be the natural projection. The induced utility plane of a bisupport $(A, T)$ for a utility $u \in \mathbb{R}^{\mathbf{A} \times \mathbf{T}}$ is the affine hull of utility vectors $u^{a}, a \in A$, extended along the utility directions of Sender types that does not use the message that this bisupport represents, i.e,

$$
\phi_{T}^{-1} \phi_{T}\left(H\left(u^{A}\right)\right)
$$

where $u^{A}=\left\{u^{a}: a \in A\right\}$. The induced utility plane of a configuration $\mathcal{C}$ for a utility $u$, denoted by $P(\mathcal{C} ; u)$, is the intersection of induced utility planes of bisupports in $\mathcal{C}$. Obviously, these induced utility planes are affine subspaces of $\mathbb{R}^{\mathrm{T}}$.

The induced utility plane of a bisupport $(A, T)$ for $u$ is the set of expectation vectors such that the utility levels of types in $T$ can be realized by a response mixed over $A$. Therefore, the induced utility plane of a configuration $\mathcal{C}$ for $u$ contains the set of all expectation vectors that satisfy optimality condition (SO) with respect to $\mathcal{C}$. Hence, by investigating the size of the induced utility plane of a configuration, we can check whether it may satisfy ( $\mathrm{SO}$ ) and if it does, how large the set of expectation vectors that satisfy (SO) may be. This is done extensively in section 4 .

For a generic $u$, the dimension of the induced utility plane of each bisupport $(A, T)$ in $\mathcal{C}$ is ${ }^{\#} A+\# T^{c}-1$ as long as it does not exceed $\#^{T}$, where $T^{c}$ is the complement of $T$ in $\mathbf{T}$. We define the $\chi$-value of a configuration to depict the typical dimension of the intersection of arbitrarily chosen affine subspaces of these dimensions in $\mathbb{R}^{\mathrm{T}}$.

Definition 2.9: For a configuration $\mathcal{C}$ on $\{\mathbf{A}, \mathbf{T}\}$, define

$$
\chi(\mathcal{C} ; \mathbf{T}) \equiv \sum_{(A, T) \in \mathcal{C}}\left({ }^{\#} A+{ }^{\#} T^{c}-1\right)-\left({ }^{\#} \mathcal{C}-1\right)^{\#} \mathbf{T}=\sum_{(A, T) \in \mathcal{C}}\left({ }^{\#} A-{ }^{\#} T\right)+\# \mathbf{T}-{ }_{\mathcal{C}}
$$

$A$ configuration $\mathcal{C}$ is said to be unstretched if

$$
\#\left(\cap_{\mathcal{C}} A\right)+\# \mathbf{T}-\#\left(\cup_{\mathcal{C}} T\right) \leq \max \{\chi(\mathcal{C} ; \mathbf{T})+1,0\}
$$

$A$ configuration $\mathcal{C}$ is said to be taut if (2.e) is fulfilled as an equality of a strictly positive number. If $\mathcal{C}$ is unstretched otherwise, it is said to be loose. If $(2 . e)$ is violated, $\mathcal{C}$ is said to be stretched. A configuration $\mathcal{C}$ is trim if every subconfiguration of $\mathcal{C}$ is unstretched. 
The induced utility plane of each bisupport in a configuration $\mathcal{C}$ contains

$\phi_{\left(\cup_{\mathcal{C}} T\right)}^{-1} \phi_{\left(\cup_{\mathcal{c}} T\right)}\left(H\left(u^{\left(\cap_{\mathcal{C}} A\right)}\right)\right)$, the affine hull of utility vectors of actions in $\cap_{\mathcal{C}} A$ extended along the utility directions of Sender types in $\left(\cup_{\mathcal{C}} T\right)^{c}$. Roughly said, a configuration is stretched if this affine subspace is generically of higher dimension than the above mentioned typical dimension ( $\chi$-value). A configuration is taut if this affine subspace is generically of the typical dimension.

It turns out that a just configuration is classified into one of the four categories set before, depending on the $\chi$-value and whether it is trim. These criteria are summarized below and developed in the rest of the paper.

Criteria for Categorization: Given a game form $(\mathbf{A}, \mathbf{T}, \pi)$, let $\mathcal{C}$ be a just configuration on $\{\mathbf{A}, \mathbf{T}\}$.

(C1) If $\mathcal{C}$ is not trim, it is in category (I).

(C2) If $\mathcal{C}$ is trim and $\chi(\mathcal{C})<0$, it is in category (II).

(C3) If $\mathcal{C}$ is trim and $\chi(\mathcal{C})>0$, it is in category (III).

(C4) If $\mathcal{C}$ is trim and $\chi(\mathcal{C})=0$, it is in category (IV).

Since there are a finite number of just configurations, generic finiteness of reduced form equilibria immediately follows from this criteria.

\section{A Preliminary Discussion}

Before going into the technical details of the proof of the main theorem, subsection 3.1 presents an illustration of the basic idea in a simple 2-type case. Subsection 3.2 discusses some basic results about semi-algebraic sets.

\subsection{A simple two-type case}

To have basic idea of the proof of the main theorem in a simple context, let us look at the 2-type case. Let $(\mathbf{A},\{1,2\}, \pi)$ be a given game form. According to Remark 2.8, we only need to consider equilibria in just configurations.

Consider equilibria in a configuration consisting of only one bisupport. This means that only one message is used and the posterior belief induced by this message is $\pi$. For 
generic utilities for the Receiver, however, there is a unique pure best response for $\pi$. Hence, there is a unique equilibrium in a configuration of this kind.

Next, consider equilibria in a configuration consisting of two or more bisupports. Recall that the messages represented by bisupports that we consider are of the following kinds; (class 1) messages sent by one type and responded by a pure action, (class 2) messages sent by both types and responded by a pure action, and (class 3 ) messages sent by both types and responded by a mixed response over two actions. The induced utility plane of these three kinds of bisupports are of the following form in $\mathbb{R}^{2}$ (the space of expectation vectors of the Sender), respectively; a vertical or horizontal line through the utility vector of a pure action, the utility vector of a pure action itself, and a straight line through two utility vector of two pure actions.

From this sketchy description of the induced utility planes, it is easy to see that the intersection of the induced utility planes of two distinct bisupports is generically at most a singleton, and that of three distinct bisupports is generically empty unless these three bisupports share a common action. If three bisupports share a common action, say $a$, the intersection of induced utility planes is generically the utility vector of $a$ itself. This means that the responses to these three messages is identical (the pure response of playing $a$ ) so that every equilibrium in this configuration (if exists) is not in reduced form. Therefore, generically there is no reduced form equilibrium in configurations with three or more distinct bisupports.

It remains to consider configurations with two bisupports. There are two cases. One case is one bisupport of the configuration represents a message in (class 2); sent by both types but responded by a pure action, say $b$. In this case, the induced utility plane of this bisupport is the utility vector of the action $b$ itself and the intersection of induced utility planes is generically empty unless $b$ is shared by both bisupports. But if $b$ is shared, the responses to these messages are identical and every equilibrium in this configuration (if exists) is not in reduced form. The other case is both bisupports of the configuration represent messages in (class 1 ) or (class 3 ). Then, the intersection of induced utility planes is generically a singleton, say $u^{*}=\left(u_{1}^{*}, u_{2}^{*}\right)$. If there is an equilibrium in this configuration, the equilibrium expected utility of the Sender is $u^{*}$. For each bisupport, 
generically, there is a unique response that is consistent $u^{*}$ in the following sense; the response mixes over the action component and induces expected utility $u_{t}^{*}$ to type $t$ Sender if $t$ is in the type component of the bisupport. It still remains to check how many different talking strategies are consistent with this configuration. A message in (class 1) obviously generates a trivial posterior concentrated on the type that sends the message. For generic utilities, the posterior generated by a message in (class 3 ) is unique because two actions are indifferent only for one posterior. Hence, for generic utilities, the two posteriors generated by the two messages used in this kind of configuration are distinct and uniquely determined so that there is at most a unique talking strategy that implements this pair of posteriors given the prior. Conclusively, for generic utilities, there is at most one pair of talking and action strategies that satisfies ( $\mathrm{SO}$ ) and (RO) with respect to this kind of configuration.

Since there are a finite number of different configurations consisting of two distinct bisupports, we showed for two type case that there are at most a finite number of reduced from equilibria for generic utilities. Observe that the analysis depended on the common actions and the dimension of induced utility planes. This idea will be generalized to set criteria to categorize configurations.

\subsection{Semi-algebraic sets}

A semi-algebraic set is a finite union of sets in $\mathbb{R}^{n}$ of the form

$$
\left\{x \in \mathbb{R}^{n}: P(x)=0 \text { and } Q_{1}(x)>0 \text { and } \ldots \text { and } Q_{J}(x)>0\right\}
$$

where $P$ and $Q_{1}, \ldots, Q_{J}$ are polynomials in $x_{1}, \ldots, x_{n}$ with real coefficients. ${ }^{(10)}$ Since a weak inequality relation is a union of an equality relation and a strict inequality relation, semi-algebraic sets may be defined by weak inequalities as well. It is clear from the definition that the class of semi-algebraic sets is closed under finite unions and intersections, complementation and (topological) closure. Remark 3.1 explains a guide to identify semialgebraic subsets for our purpose, and Lemma 3.2 presents a criterion for a semi-algebraic subset $S$ to be negligible (equivalently, for the complement of $S$ to be generic).

(10) Notice that $\left[P_{1}(x)=0\right.$ or $\ldots$ or $\left.P_{j}(x)=0\right]$ is equivalent to $P_{1}(x) \ldots P_{j}(x)=0$ and $\left[P_{1}(x)=\right.$ 0 and $\ldots$ and $\left.P_{j}(x)=0\right]$ is equivalent to $P_{1}(x)^{2}+\cdots+P_{j}(x)^{2}=0$, so that these conditions can be represented as one polynomial set equal to 0 . 
Remark 3.1: The Tarski-Seidenberg theorem asserts that every first order formula is equivalent to a first order formula without quantifiers. According to this theorem, a subset of $\mathbb{R}^{n}$ is semi-algebraic if it is defined by polynimial (in)equalities supplemented by quantifiers. (The implication of the Tarski-Seidenberg theorem is far more powerful than this. See Benedetti and Risler (1990) for the Tarski-Seidenberg theorem and its exploitation.) The subsets of utilities of importance in this paper admit the following characterization: there exist (exactly) a certain number of linearly independent vectors (these are quantifiers) determined by the utility through polynomial inequalities. Since certain determinants vanishing or not is equivalent to certain polynomial (in)equalities, these subsets of utilities are semi-algebraic.

Lemma 3.2: A semi-algebraic set $S$ in $\mathbb{R}^{n}$ is negligible if and only if the interior of $S$ is empty.

Proof: This is an immediate corollary of Whitney (1957) which basically asserts that every semi-algebraic set is decomposed into a finite number of disjoint, connected semi-algebraic manifolds. (See also Benedetti and Risler (1990) for stratification.)

We close this section by identifying a certain class of functions that maps a generic set to a generic set.

Lemma 3.3: Let $f: X \rightarrow Y$ be a surjective submersion that maps any semi-algebraic set to a semi-algebraic set. Then, $f(G) \subset Y$ is generic if $G \subset X$ is generic.

Proof: We need to show that the complement of $f(G)$ is negligible. Since $f(G)$ is semialgebraic, it suffices to show that the complement has no interior. Suppose on the contrary that $Z$ is a nonempty open set contained in the complement of $f(G)$. Then, $f^{-1}(Z)$ is contained in the complement of $G$. Moreover, since $f$ is continuous and surjective, $f^{-1}(Z)$ is a nonempty open set. This is a contradiction to $G$ being generic.

Remark 3.4: The condition in Lemma 3.3 that $F$ maps any semi-algebraic set to a semialgebraic set, is needed because we required a generic subset to be semi-algebraic. In fact, Lemma 3.3 is applied later to rational functions. 


\section{Generic Dimension of the Induced Utility Plane}

This section investigates the generic dimension of the induced utility plane of various sorts of configurations. In light of Remark 2.8, only just configurations are considered. As discussed in subsection 4.3, the results in this section establish (C1), (C2) and a somewhat weaker version of (C4) in the Criteria of Categorization as stated at the end section 2. Complete establishment of the Criteria is done in section 5.

Configurations are classified as in Definition 4.1, and the results are established for a special class of configurations first and are extended to more general classes.

Definition 4.1 : A configuration $\mathcal{C}$ on $\{\mathbf{A}, \mathbf{T}\}$ is cacophonous if $T=\mathbf{T}$ for all $(A, T) \in \mathcal{C}$. $A$ configuration $\mathcal{C}$ is annexed if $\cap_{\mathcal{C}} A \neq \emptyset$.

A cacophonous configuration is generated by an equilibrium in which every type uses every message and an annexed configuration is generated by an equilibrium in which some particular action is used in response to every message.

Subsection 4.1 investigates generic dimensions of annexed configurations; results are proved for cacophonous configurations first and then extended to all annexed configurations. Based on these results, non-annexed configurations are investigated in subsection 4.2. For conciseness of expression, a notation is introduced.

Notation : Let $\mathcal{W}$ be a vector space and $Q(w)$ and $R(w)$ be functions of $w \in \mathcal{W}$. The notation " $Q(w) \frac{w}{\mathcal{W}} R(w)$ " means "there is a generic subset $G$ of $\mathcal{W}$ such that $Q(w)=R(w)$ for all $w \in G$."

\subsection{Annexed configurations}

Generic characterization of induced utility planes of annexed configurations is as follows.

Proposition 4.2 : Given a game form $(\mathbf{A}, \mathbf{T}, \pi)$, let $\mathcal{C}$ be a just and annexed configuration on $\{\mathbf{A}, \mathbf{T}\}$. Suppose that every proper subconfiguration of $\mathcal{C}$ is unstretched. Let $\mathcal{U}=$ $\mathbb{R}^{\mathbf{A} \times \mathbf{T}}$.

(a) If $\mathcal{C}$ is trim, $\operatorname{dim} P(\mathcal{C} ; u) \frac{u}{\bar{u}} \chi(\mathcal{C} ; \mathbf{T})$.

(b) If $\mathcal{C}$ is stretched, $P(\mathcal{C} ; u) \frac{u}{u} H\left(u^{\left(\cap_{c} A\right)}\right)$. 
Remark 4.3 : Let $a_{0} \in \cap_{\mathcal{C}} A$. Since the utility of each Sender type is invariant under addition of a constant, we may fix the utility vector of $a_{0}$ to be 0 . Hence, Proposition 4.2 with $\mathcal{U}$ replaced by $\mathcal{U}_{0}=\left\{u \in \mathcal{U}: u^{a_{0}}=0\right\}$, is an equivalent statement.

The induced utility plane of an annexed configuration always contains the utility vector of a common action, say $a_{0}$. Conceptually, this utility vector can be regarded as the origin of the space of expectation vectors as explained in Remark 4.3. If a just configuration $\mathcal{C}$ is cacophonous as well as annexed, investigation of its induced utility plane is easier: the induced utility plane of $(A, \mathrm{~T})$ for $u$ is simply $H\left(u^{A}\right)$, and $\mathcal{C}$ is unstretched if $\#\left(\cap_{\mathcal{C}} A\right) \leq \chi(\mathcal{C} ; \mathbf{T})+1$. (Recall $\cup_{\mathcal{C}} T=\mathbf{T}$.) Hence, Proposition 4.2 is proved first for cacophonous configurations.

The proof of the proposition for cacophonous configurations uses a double induction: the primary induction is on the cardinality of configuration and within each primary induction, secondary induction is employed on the smallest action component of all bisupports. In the induction process, we derive information on the given configuration by investigating certain variants of the given configuration. Next two Lemmas provide preliminary results on these variants.

Lemma 4.4 : Let $\mathcal{C}$ be a just and cacophonous configuration on $\{\mathbf{A}, \mathbf{T}\}$.

i) If $\mathcal{C}=\mathcal{C}^{\prime} \cup \mathcal{C}^{\prime \prime}$ and $\mathcal{C}^{\prime} \cap \mathcal{C}^{\prime \prime}=\emptyset$, then $\chi(\mathcal{C} ; \mathbf{T})=\chi\left(\mathcal{C}^{\prime} ; \mathbf{T}\right)+\chi\left(\mathcal{C}^{\prime \prime} ; \mathbf{T}\right)-\# \mathbf{T}$.

ii) If $A^{0}=\cap_{\mathcal{C}} A$ for some $\left(A^{0}, \mathbf{T}\right) \in \mathcal{C}$, then $\mathcal{C}$ is stretched.

Proof : Part $i$ ) is immediate from formula (2.d), the definition of $\chi$-value. For a configuration considered in part $i i)$, the left hand side of $(2 . d)$ is $\# A^{0}>0$. By $\left.i\right), \chi(\mathcal{C} ; \mathbf{T})=$ $\chi\left(\mathcal{C} \backslash\left\{\left(A^{0}, \mathbf{T}\right)\right\} ; \mathbf{T}\right)+\chi\left(\left\{\left(A^{0}, \mathbf{T}\right)\right\} ; \mathbf{T}\right)-\# \mathbf{T}$. Because $\mathcal{C}$ is just, $\chi\left(\mathcal{C} \backslash\left\{\left(A^{0}, \mathbf{T}\right)\right\} ; \mathbf{T}\right)<\# \mathbf{T}$ so that $\chi(\mathcal{C} ; \mathbf{T})<\# A^{0}-1$. So, $(2 . e)$ is violated and $\mathcal{C}$ is stretched.

Lemma 4.5: Let $\mathcal{C}$ be a just configuration on $\{\mathbf{A}, \mathbf{T}\}$ which is annexed and cacophonous. Suppose that every proper subconfiguration of $\mathcal{C}$ is unstretched.

(a) Suppose that a proper non-singleton subconfiguration $\mathcal{C}^{\prime}$ is taut. Let $A^{\prime}=\cap_{\mathcal{C}^{\prime}} A$ and consider a new configuration $\mathcal{C}^{*}=\left(\mathcal{C} \backslash \mathcal{C}^{\prime}\right) \cup\left\{\left(A^{\prime}, \mathbf{T}\right)\right\}$. Then,

i) $\chi\left(\mathcal{C}^{*} ; \mathbf{T}\right)=\chi(\mathcal{C} ; \mathbf{T})$

ii) $\mathcal{C}^{*}$ is unstretched if and only if $\mathcal{C}$ is unstretched, and 
iii) every proper subconfiguration of $\mathcal{C}^{*}$ is unstretched.

(b) Suppose that every proper non-singleton subconfiguration of $\mathcal{C}$ is loose and that there is a bisupport $\left(A^{0}, \mathbf{T}\right) \in \mathcal{C}$ such that $\# A^{0} \geq 2$. Let $\tilde{A}^{0}=A^{0} \backslash\{b\}$ for some $b \in A^{0}$, and consider a new configuration $\tilde{\mathcal{C}}=\left(\mathcal{C} \backslash\left\{\left(A^{0}, \mathbf{T}\right)\right\}\right) \cup\left\{\left(\tilde{A}^{0}, \mathbf{T}\right)\right\}$. Then, every proper subconfiguration of $\tilde{\mathcal{C}}$ is unstretched. Moreover, under the further hypothesis that $b \notin \cap_{\mathcal{C}} A, \tilde{\mathcal{C}}$ is unstretched if and only if $\mathcal{C}$ is loose.

Proof: (a) Consider a subconfiguration of $\mathcal{C}^{*}$ of the form $\mathcal{C}^{\prime \prime} \cup\left\{\left(A^{\prime}, \mathbf{T}\right)\right\}$ where $\mathcal{C}^{\prime \prime} \subset \mathcal{C} \backslash \mathcal{C}^{\prime}$. Since $\mathcal{C}^{\prime}$ is taut, $\chi\left(\mathcal{C}^{\prime} ; \mathbf{T}\right)=\# A^{\prime}-1=\chi\left(\left\{\left(A^{\prime}, \mathbf{T}\right)\right\} ; \mathbf{T}\right)$. Therefore, by Lemma 4.4 (a),

$$
\chi\left(\mathcal{C}^{\prime \prime} \cup\left\{\left(A^{\prime}, \mathbf{T}\right)\right\} ; \mathbf{T}\right)=\chi\left(\mathcal{C}^{\prime \prime} ; \mathbf{T}\right)+\chi\left(\mathcal{C}^{\prime} ; \mathbf{T}\right)-{ }^{\# \mathbf{T}}=\chi\left(\mathcal{C}^{\prime \prime} \cup \mathcal{C}^{\prime} ; \mathbf{T}\right)
$$

Moreover, since $A^{\prime}=\cap_{\mathcal{C}^{\prime}} A$,

$$
\cap_{\left(\mathcal{C}^{\prime \prime} \cup\left\{\left(A^{\prime}, \mathbf{T}\right)\right\}\right)} A=\cap_{\left(\mathcal{C}^{\prime \prime} \cup \mathcal{C}^{\prime}\right)} A
$$

Taking $\mathcal{C}^{\prime \prime}=\mathcal{C} \backslash \mathcal{C}^{\prime},(4 . a)$ immediately proves (a)-i).

Since both $\mathcal{C}$ and $\mathcal{C}^{*}$ are apparently annexed and cacophonous, a subconfiguration of either of these is unstretched if the number of common actions is at most the $\chi$-value plus 1 . Therefore, by (4.a) and (4.b),

$$
\mathcal{C}^{\prime \prime} \cup\left\{\left(A^{\prime}, \mathbf{T}\right)\right\} \text { is unstretched if and only if } \mathcal{C}^{\prime \prime} \cup \mathcal{C}^{\prime} \text { is unstretched. }
$$

Taking $\mathcal{C}^{\prime \prime}=\mathcal{C} \backslash \mathcal{C}^{\prime},(4 . c)$ immediately proves (a)-ii).

To prove (a)-iii), take a proper subconfiguration of $\mathcal{C}^{*}$. Since a singleton configuration trivially is unstretched by definition, we consider subconfigurations consisting of two or more bisupports. If it does not contain $\left(A^{\prime}, \mathbf{T}\right)$, it is a proper subconfiguration of $\mathcal{C}$ so it is unstretched. If it contains $\left(A^{\prime}, \mathbf{T}\right)$, it is of the form $\mathcal{C}^{\prime \prime} \cup\left\{\left(A^{\prime}, \mathbf{T}\right)\right\}$ where $\mathcal{C}^{\prime \prime}$ is a proper subset of $\mathcal{C} \backslash \mathcal{C}^{\prime}$. So $\mathcal{C}^{\prime \prime} \cup \mathcal{C}^{\prime}$ is a proper subconfiguration of $\mathcal{C}$ and is unstretched by hypothesis. Therefore, $\mathcal{C}^{\prime \prime} \cup\left\{\left(A^{\prime}, \mathbf{T}\right)\right\}$ is unstretched by (4.c).

(b) Consider $\tilde{\mathcal{C}}$ as described in part (b) and a proper subconfiguration $\tilde{\mathcal{C}}^{\prime \prime}$. Let $\mathcal{C}^{\prime \prime}$ be the proper subconfiguration of $\mathcal{C}$ obtained from $\tilde{\mathcal{C}}^{\prime \prime}$ by replacing $\left(\tilde{A}^{0}, \mathbf{T}\right)$ with $\left(A^{0}, \mathbf{T}\right)$. Then,

$$
\#\left(\cap_{\tilde{\mathcal{C}}^{\prime \prime}} A\right) \leq \#\left(\cap_{\mathcal{C}^{\prime \prime}} A\right) \text { and } \chi\left(\tilde{\mathcal{C}}^{\prime \prime}\right)=\chi\left(\mathcal{C}^{\prime \prime} ; \mathbf{T}\right)-1
$$


Since $\mathcal{C}$ is annexed, $\cap_{\mathcal{C}^{\prime \prime}} A \neq \emptyset$ so that $\mathcal{C}^{\prime \prime}$ being loose implies $\#\left(\cap_{\mathcal{C}^{\prime \prime}} A\right) \leq \chi\left(\mathcal{C}^{\prime \prime} ; \mathbf{T}\right)$. By (4.d), \#( $\left.\cap_{\tilde{\mathcal{C}}^{\prime \prime}} A\right) \leq \chi\left(\tilde{\mathcal{C}}^{\prime \prime} ; \mathbf{T}\right)+1$, which implies that $\tilde{\mathcal{C}}^{\prime \prime}$ is unstretched.

If $b \notin \cap_{\mathcal{C}} A$, then $\cap_{\tilde{\mathcal{C}}} A=\cap_{\mathcal{C}} A$ while $\chi(\tilde{\mathcal{C}} ; \mathbf{T})=\chi(\mathcal{C} ; \mathbf{T})-1$. Hence, if $\mathcal{C}$ is loose, $\tilde{\mathcal{C}}$ is unstretched. If $\mathcal{C}$ is stretched or is taut, $\tilde{\mathcal{C}}$ is stretched.

Proof of Proposition 4.2 for cacophonous configurations : Recall the induced utility plane of $(A, \mathbf{T})$ is $H\left(u^{A}\right)$ so that $P(\mathcal{C} ; u)=\cap_{\mathcal{C}} H\left(u^{A}\right)$. Let $A^{0}$ be a smallest action component, that is, ${ }^{A} A \geq \#_{A^{0}}$ for all $(A, \mathbf{T}) \in \mathcal{C}$. Let $\mathcal{C}_{-A^{0}}=\mathcal{C} \backslash\left\{\left(A^{0}, \mathbf{T}\right)\right\}$.

Step 1: $\# \mathcal{C}=1$, i.e, $\mathcal{C}=\left\{\left(A^{0}, \mathbf{T}\right)\right\}$

In this case, $\mathcal{C}$ is trivially trim and $H\left(u^{A^{0}}\right) \frac{u}{\mathcal{U}}\left(\# A^{0}-1\right)=\chi(\mathcal{C} ; \mathbf{T})$ is immediate.

Step 2: $\quad 2 \leq \#_{\mathcal{C}} \leq \#_{\mathrm{T}}+1$ and $\#_{A^{0}=1}$.

By Lemma 4.4 (b), $\mathcal{C}$ is stretched. Clearly, $H\left(u^{\left(\cap_{\mathcal{C}} A\right)}\right) \subset \cap_{\mathcal{c}} H\left(u^{A}\right) \subset H\left(u^{A^{0}}\right)$. Since $\cap_{\mathcal{C}} A=A^{0}, \cap_{\mathcal{C}} H\left(u^{A}\right)=H\left(u^{\left(\cap_{\mathcal{c}} A\right)}\right)$ for all $u \in \mathcal{U}$.

Step 3: $\quad 2 \leq \# \mathcal{C} \leq \# \mathrm{~T}+1$ and $1<\#_{A^{0}} \leq \#_{\mathrm{T}}$.

(Case 1) Consider the case that a proper non-singleton subconfiguration $\mathcal{C}^{\prime}$ of $\mathcal{C}$ is taut. Let $A^{\prime}=\cap_{\mathcal{C}^{\prime}} A$. Then, $\cap_{\mathcal{C}^{\prime}} H\left(u^{A}\right) \frac{u}{\bar{u}} H\left(u^{A^{\prime}}\right)$ by induction hypothesis. Therefore,

$$
P(\mathcal{C} ; u) \stackrel{u}{\frac{u}{u}} P\left(\mathcal{C}^{*} ; u\right) \text { where } \mathcal{C}^{*}=\left(\mathcal{C} \backslash \mathcal{C}^{\prime}\right) \cup\left\{\left(A^{\prime}, \mathbf{T}\right)\right\}
$$

If $\mathcal{C}$ is unstretched, by Lemma $4.5(\mathrm{a}), \chi(\mathcal{C} ; \mathbf{T})=\chi\left(\mathcal{C}^{*} ; \mathbf{T}\right)$ and $\mathcal{C}^{*}$ satisfies conditions of Proposition 4.2 (a) so that $\operatorname{dim} P\left(\mathcal{C}^{*} ; u\right) \frac{u}{\bar{u}} \chi(\mathcal{C} ; \mathbf{T})$ by induction hypothesis. By (4.e), we are done. If $\mathcal{C}$ is stretched, analogous reasoning leads to $P\left(\mathcal{C}^{*} ; u\right) \frac{u}{\bar{u}} H\left(u^{\left(\cap^{*} A\right)}\right)$. From (4.e) and the fact $\cap_{\mathcal{C}^{*}} A=\cap_{\mathcal{C}} A$, we conclude $P(\mathcal{C} ; u) \frac{u}{\bar{u}} H\left(u^{\left(\cap_{\mathcal{C}} A\right)}\right)$.

(Case 2) Consider the case that every proper non-singleton subconfiguration of $\mathcal{C}$ is loose. If $A^{0}=\cap_{\mathcal{C}} A, \mathcal{C}$ is stretched by Lemma $4.4(\mathrm{~b})$, and $P(\mathcal{C} ; u)=H\left(u^{\left(\cap_{\mathcal{C}} A\right)}\right)$ by the same reasoning as in Step 2 . In the rest of the proof, we assume $A^{0} \neq \cap_{\mathcal{C}} A$.

Let $\tilde{\mathcal{C}}$ be the new configuration obtained from $\mathcal{C}$ by replacing $\left(A^{0}, \mathbf{T}\right)$ with $\left(\tilde{A}^{0}, \mathbf{T}\right)$ where $\tilde{A}^{0}=A^{0} \backslash\{b\}$ for some $b \in A^{0}$. Since $A^{0} \neq \cap_{\mathcal{C}} A$, we assume $b \notin \cap_{\mathcal{C}} A$. Then, by Lemma 4.5 (b), every proper subconfiguration of $\tilde{\mathcal{C}}$ is unstretched. We know, by induction hypothesis, about the generic dimension of $P(\tilde{\mathcal{C}} ; u)$. We will investigate what would happen if we add $b$ back to $\tilde{A}^{0}$. 
If $\mathcal{C}$ is loose, $\tilde{\mathcal{C}}$ is unstretched by Lemma 4.5 (b) so that $\operatorname{dim} P(\tilde{\mathcal{C}} ; u) \frac{u}{\bar{u}} \chi(\tilde{\mathcal{C}} ; \mathbf{T})$. In fact, $P(\tilde{\mathcal{C}} ; u)$ is the intersection of $P\left(\mathcal{C}_{-A^{0}} ; u\right)$ and $H\left(u^{\tilde{A}^{0}}\right)$ whose generic dimensions are $\chi\left(\mathcal{C}_{-A^{0}} ; \mathbf{T}\right)$ and $\#_{A^{0}}-2$, respectively. Since $P(\tilde{\mathcal{C}} ; u) \neq \emptyset$ (notice $\tilde{\mathcal{C}}$ is still annexed), simple linear algebra asserts

$$
\operatorname{dim} H\left(P\left(\mathcal{C}_{-A^{0}} ; u\right) \cup H\left(u^{\tilde{A}^{0}}\right)\right) \stackrel{u}{=} \chi\left(\mathcal{C}_{-A^{0}} ; \mathbf{T}\right)+\left({ }^{\#} A^{0}-2\right)-\chi(\tilde{\mathcal{C}})={ }^{\#} \mathbf{T}
$$

or equivalently, $H\left(P\left(\mathcal{C}_{-A^{0}} ; u\right) \cup H\left(u^{\tilde{A}^{0}}\right)\right) \frac{u}{\bar{u}} \mathbb{R}^{\mathbf{T}}$. This means that the affine hull of the union of $P\left(\mathcal{C}_{-A^{0}} ; u\right)$ and $H\left(u^{A^{0}}\right)$ is also $\mathbb{R}^{\mathrm{T}}$ generically. Since $\operatorname{dim} H\left(u^{A^{0}}\right) \frac{u}{\bar{u}} \#^{0}-1$, we have $\operatorname{dim} P(\mathcal{C} ; u)=\operatorname{dim}\left(P\left(\mathcal{C}_{-A^{0}} ; u\right) \cap H\left(u^{A^{0}}\right)\right) \frac{u}{\bar{u}} \chi\left(\mathcal{C}_{-A^{0}} ; \mathbf{T}\right)+\left({ }^{\#} A^{0}-1\right)-\# \mathbf{T}=\chi(\mathcal{C} ; \mathbf{T})$.

To analyze the case that $\mathcal{C}$ is taut or stretched, we establish two Lemmas. First, partition $\mathcal{C}_{-A^{0}}$ into two parts; $\mathcal{C}_{-A^{0}}=\mathcal{C}^{b} \cup \mathcal{C}^{-b}$ where $\mathcal{C}^{b}$ is the set of bisupports in $\mathcal{C}_{-A^{0}}$ that contain $b$ (that is, $\left.\mathcal{C}^{b}=\left\{(A, \mathbf{T}) \in \mathcal{C}_{-A^{0}}: \quad b \in A\right\}\right)$, and $\mathcal{C}^{-b}$ is the complement of $\mathcal{C}^{b}$ in $\mathcal{C}_{-A^{0}}$. To facilitate discussion, we introduce notation: given $u \in \mathcal{U}$ and $x \in \mathbb{R}^{\mathbf{T}}$, let $\left.u\right|_{(x \backslash b)}$ denote the utility obtained from $u$ by substituting $x$ for $u^{b}{ }^{(11)}$

Lemma 4.6 : If $\mathcal{C}^{b} \neq \emptyset$, there exists a generic subset $G$ of $\mathcal{U}$ such that, for all $u \in G$, $P\left(\mathcal{C}^{b} ; u\right) \underset{P\left(\overline{\mathcal{C}^{b}} ; u\right)}{\frac{x}{}} P\left(\mathcal{C}^{b} ;\left.u\right|_{(x \backslash b)}\right)$.

Proof : Take a bisupport $(A, \mathbf{T}) \in \mathcal{C}^{b}$ and let $G_{(A, \mathbf{T})}$ denote the generic subset of $\mathcal{U}$ for which the induced utility planes of the following configurations have the generic dimensions as determined by induction hypothesis; $\mathcal{C}^{b}$ and $\hat{\mathcal{C}}^{b}=\left(\mathcal{C}^{b} \backslash\{(A, \mathbf{T})\}\right) \cup\{(A \backslash\{b\}, \mathbf{T})\}$.

Every point $x$ in $H\left(u^{A}\right) \backslash H\left(u^{A \backslash\{b\}}\right)$ is in the affine hull of $H\left(u^{A \backslash\{b\}}\right)$ and $u^{b}$. Conversely, $u^{b}$ is in the affine hull of $H\left(u^{A \backslash\{b\}}\right)$ and $x$. Therefore,

$$
H\left(u^{A}\right)=H\left(\left(\left.u\right|_{(x \backslash b)}\right)^{A}\right) \text { for all } x \in H\left(u^{A}\right) \backslash H\left(u^{A \backslash\{b\}}\right)
$$

Since it is assumed that $b \notin \cap_{\mathcal{C}} A, \hat{\mathcal{C}}^{b}$ is trim. Hence, for $u \in G_{(A, \mathbf{T})}, \operatorname{dim} P\left(\hat{\mathcal{C}}^{b} ; u\right)=$ $\operatorname{dim} P\left(\mathcal{C}^{b} ; u\right)-1$ so that $P\left(\mathcal{C}^{b} ; u\right) \backslash P\left(\hat{\mathcal{C}}^{b} ; u\right)$ is a generic subset of $P\left(\mathcal{C}^{b} ; u\right)$. It is clear that $\left(P\left(\mathcal{C}^{b} ; u\right) \backslash P\left(\hat{\mathcal{C}}^{b} ; u\right)\right) \subset\left(H\left(u^{A}\right) \backslash H\left(u^{A \backslash\{b\}}\right)\right)$. Therefore, by $(4 . f)$,

$$
\left[u \in G_{(A, \mathbf{T})}\right] \Rightarrow\left[H\left(u^{A}\right) \underset{P\left(\mathcal{C}^{b} ; u\right)}{\stackrel{x}{=}} H\left(\left(\left.u\right|_{(x \backslash b)}\right)^{A}\right)\right]
$$

(11) That is, $\left.u\right|_{(x \backslash b)} \in \mathcal{U}$ such that $\left(\left.u\right|_{(x \backslash b)}\right)^{b}=x$ and $\left(\left.u\right|_{(x \backslash b)}\right)^{b^{\prime}}=u^{b^{\prime}}$ for all $b^{\prime} \neq b$. 
Since this is true for each bisupport, $P\left(\mathcal{C}^{b} ; u\right) \underset{P\left(\overline{\left.\mathcal{C}^{b} ; u\right)}\right.}{\frac{x}{}} P\left(\mathcal{C}^{b} ;\left.u\right|_{(x \backslash b)}\right)$ if $u \in \cap_{\mathcal{C}}\left(G_{(A, T)}\right)$. As a finite intersection of generic sets, $\cap_{\mathcal{C}}\left(G_{(A, T)}\right)$ is generic in $\mathcal{U}$ and the proof is complete.

Lemma 4.7 : Suppose that $\operatorname{dim} H\left(P\left(\mathcal{C}_{-A^{0}} ; u\right) \cup H\left(u^{\tilde{A}^{0}}\right)\right)<\# \mathbf{T}$ for generic $u$. Then, $u^{b} \notin H\left(P\left(\mathcal{C}_{-A^{0}} ; u\right) \cup H\left(u^{\tilde{A}^{0}}\right)\right)$ for generic $u$.

Proof : For notational simplicity, let $\mathcal{H}(u)=H\left(P\left(\mathcal{C}_{-A^{0}} ; u\right) \cup H\left(u^{\tilde{A}^{0}}\right)\right)$. If $\mathcal{C}^{b}=\emptyset, \mathcal{H}(u)$ is determined independently of $u^{b}$ and the conclusion is immediate. Next, suppose $\mathcal{C}^{b} \neq \emptyset$ and consider the set $\mathcal{U}_{\mathcal{H}}$ of utilities for which $u^{b} \in \mathcal{H}(u)$. This set is semi-algebraic by Remark 3.1. In light of Lemma 3.2, we will show that $\mathcal{U}_{\mathcal{H}}$ is negligible by showing that it has empty interior.

To reach a contradiction, suppose $u$ is an interior point of $\mathcal{U}_{\mathcal{H}}$. Consider the configuration $\mathcal{C}^{b} \cup\left\{\left(\tilde{A}^{0}, \mathbf{T}\right)\right\}$. This configuration is trim because $\mathcal{C}^{b} \cup\left\{\left(A^{0}, \mathbf{T}\right)\right\}$ is loose as a proper subconfiguration of $\mathcal{C}$. Hence, the affine hull of the induced utility plane of $\mathcal{C}^{b}$ and that of $\left(\tilde{A}^{0}, \mathbf{T}\right)$ has generic dimension $\chi\left(\mathcal{C}^{b} ; \mathbf{T}\right)+\left({ }^{\#} A^{0}-2\right)-\chi\left(\mathcal{C}^{b} \cup\left\{\left(\tilde{A}^{0}, \mathbf{T}\right)\right\} ; \mathbf{T}\right)=\# \mathbf{T}$. Hence, we may assume $H\left(P\left(\mathcal{C}^{b} ; u\right) \cup H\left(u^{\bar{A}^{0}}\right)\right)=\mathbb{R}^{\mathbf{T}}$. On the other hand, we may assume $\mathcal{H}(u)=H\left(P\left(\mathcal{C}_{-A^{0}} ; u\right) \cup H\left(u^{\tilde{A}^{0}}\right)\right) \neq \mathbb{R}^{\mathbf{T}}$ by hypothesis. This means that $P\left(\mathcal{C}^{b} ; u\right)$ has some point outside of $\mathcal{H}(u)$, which in turn means that $P\left(\mathcal{C}^{b} ; u\right) \backslash \mathcal{H}(u)$ is a generic subset of $P\left(\mathcal{C}^{b} ; u\right)$ because we are dealing with affine subspaces.

By Lemma 4.6, we can find $x \notin \mathcal{H}(u)$ arbitrarily close to $u^{b}$ such that $P\left(\mathcal{C}^{b} ; u\right)=$ $P\left(\mathcal{C}^{b} ;\left.u\right|_{(x \backslash b)}\right)$ so that $P\left(\mathcal{C}_{-A^{0}} ; u\right)=P\left(\mathcal{C}_{-A^{0}} ;\left.u\right|_{(x \backslash b)}\right)$. Because $b \notin \tilde{A}^{0}$, this means that $\mathcal{H}\left(\left.u\right|_{(x \backslash b)}\right)=\mathcal{H}(u)$. Hence, $x=\left(\left.u\right|_{(x \backslash b)}\right)^{b} \notin \mathcal{H}\left(\left.u\right|_{(x \backslash b)}\right)$ so that $\left.u\right|_{(x \backslash b)} \notin \mathcal{U}_{\mathcal{H}}$. This contradicts $u$ being an interior point of $\mathcal{U}_{\mathcal{H}}$.

Now, return to the main proof and suppose $\mathcal{C}$ is not loose. By Lemma 4.5 (b), $\tilde{\mathcal{C}}$ is stretched and $P(\tilde{\mathcal{C}} ; u) \frac{u}{\bar{u}} H\left(u^{\left(\cap_{\mathcal{c}}^{A}\right)}\right)$ by induction hypothesis. Then, the generic dimension of $H\left(P\left(\mathcal{C}_{-A^{0}} ; u\right) \cup H\left(u^{\bar{A}^{0}}\right)\right)$ is $\chi\left(\mathcal{C}_{-A^{0}} ; \mathbf{T}\right)+\left({ }^{\#} A^{0}-2\right)-\left(\#\left(\cap_{\tilde{c}} A\right)-1\right)$ which is equal to $\chi(\mathcal{C} ; \mathbf{T})-\#\left(\cap_{\mathcal{C}} A\right)+\# \mathbf{T}$ by Lemma $4.4(\mathrm{a})$ and the fact $\cap_{\tilde{c}} A=\cap_{\mathcal{C}} A$. Since $\mathcal{C}$ is not loose, $\chi(\mathcal{C} ; \mathbf{T})<\#\left(\cap_{\mathcal{C}} A\right)$ so that $\operatorname{dim} H\left(P\left(\mathcal{C}_{-A^{0}} ; u\right) \cup H\left(u^{\tilde{A}^{0}}\right)\right)<\# \mathbf{T}$ for generic $u$. So, by Lemma 4.7,

$$
u^{b} \notin H\left(P\left(\mathcal{C}_{-A^{0}} ; u\right) \cup H\left(u^{\bar{A}^{0}}\right)\right) \text { for generic } u \text {. }
$$


Using this, we will show $P(\mathcal{C} ; u) \frac{u}{\bar{u}} P(\tilde{\mathcal{C}} ; u)$. Clearly, $P(\tilde{\mathcal{C}} ; u) \subset P(\mathcal{C} ; u)$. Suppose that $P(\mathcal{C} ; u)$ has a point $z \notin P(\tilde{\mathcal{C}} ; u)$. This means that $z \in P\left(\mathcal{C}_{-A^{0}} ; u\right)$ and $z=\beta x+$ $(1-\beta) u^{b}$ where $x \in H\left(u^{\tilde{A}^{0}}\right)$. This in turn means that $u^{b} \in H\left(P\left(\mathcal{C}_{-A^{0}} ; u\right) \cup H\left(u^{\tilde{A}^{0}}\right)\right)$. By (4.g), this is not possible generically, and we conclude that $P(\mathcal{C} ; u) \frac{u}{\bar{u}} P(\tilde{\mathcal{C}} ; u)$. Since $P(\tilde{\mathcal{C}} ; u) \frac{u}{\bar{u}} H\left(u^{\left(\cap_{\mathcal{C}} A\right)}\right)$ as discussed above, $P(\mathcal{C} ; u) \frac{u}{\bar{u}} H\left(u^{\left(\cap_{\mathcal{C}} A\right)}\right)$.

If $\mathcal{C}$ is taut, $\chi(\mathcal{C} ; \mathbf{T})={ }^{\#} \cap_{\mathcal{C}} A-1$ so that $\operatorname{dim} P(\mathcal{C} ; u) \frac{u}{\bar{u}} \chi(\mathcal{C} ; \mathbf{T})$. If $\mathcal{C}$ is stretched, there is nothing more to prove. This completes the proof of Proposition 4.2 for cacophonous configurations.

We extend the proof to annexed configurations which are not cacophonous; From the given configuration, we construct a cacophonous configuration about the induced utility plane of which we have information. Then, we find a relationship between the induced utility plane of the constructed configuration and that of the original one, which leads to the conclusion.

\section{Proof of Proposition 4.2 for configurations that are not cacophonous :}

Let $a_{0} \in \cap_{\mathcal{C}} A$. In light of Remark 4.3, we shall prove Proposition 4.2 with respect to $\mathcal{U}_{0}=\left\{u \in \mathcal{U}: u^{a_{0}}=0\right\}$.

First, construct the following configuration on $\{\mathbf{A} \cup \mathbf{T}, \mathbf{T}\}$ from $\mathcal{C}$ :

$$
\mathcal{C}^{c a}=\left\{\left(A \cup T^{c}, \mathbf{T}\right):(A, T) \in \mathcal{C}\right\}
$$

The relevant utility space for $\mathcal{C}^{c a}$ is $\mathcal{U}_{0}^{c a}=\mathcal{U}_{0} \times \mathbb{R}^{\mathbf{T} \times \mathbf{T}}$. Clearly, $\mathcal{C}^{c a}$ is annexed and cacophonous. Since $\mathcal{C}$ is in reduced form, $\#_{A} \leq \#_{T}$ for all $(A, T) \in \mathcal{C}$. Therefore, $\#_{A}+\#_{T^{c}} \leq \#_{\mathbf{T}}$ for all $\left(A \cup T^{c}, \mathbf{T}\right) \in \mathcal{C}^{c a}$, which means that $\mathcal{C}^{c a}$ is just. Moreover, it is immediate from the definition of $\chi$-value that $\chi(\mathcal{C})=\chi\left(\mathcal{C}^{c a}\right)$, and a subconfiguration of $\mathcal{C}^{c a}$ is unstretched if and only if the corresponding subconfiguration of $\mathcal{C}$ is unstretched. Therefore, by Proposition 4.2 for cacophonous configurations and Remark 4.3, we have

(1) If $\mathcal{C}$ is trim, so is $\mathcal{C}^{c a}$ and $\operatorname{dim} P\left(\mathcal{C}^{c a} ; \mathbf{u}\right) \frac{\mathbf{u}}{\overline{\mathcal{U}_{0}^{c a}}} \chi(\mathcal{C} ; \mathbf{T})$.

(2) If $\mathcal{C}$ is stretched, so is $\mathcal{C}^{c a}$ and $P\left(\mathcal{C}^{c a} ; \mathfrak{u}\right) \frac{\bar{u}}{\overline{\bar{u}_{0}^{c a}}} H\left(\mathbf{u}^{\left(\cap_{\mathcal{C}}\left(A \cup T^{c}\right)\right)}\right)$.

Suppose there is a mapping $L: \mathcal{O} \rightarrow \mathcal{U}_{0}$, where $\mathcal{O}$ is an open subset of $\mathcal{U}_{0}^{c a}$, such that 
i) $\operatorname{dim} P(\mathcal{C} ; L(\mathbf{u}))=\operatorname{dim} P\left(\mathcal{C}^{c a} ; \mathbf{u}\right)$ for all $\mathbf{u} \in \mathcal{O}$, and

ii) $L(G)$ is a generic subset of $\mathcal{U}_{0}$ if $G$ is a generic subset of $\mathcal{O}$.

Under this supposition, we are able to prove Proposition 4.2 for the considered configurations by the following arguments:

If $\mathcal{C}$ is trim, by (1) and $i), \operatorname{dim} P(\mathcal{C} ; u)=\chi(\mathcal{C} ; \mathbf{T})$ for all $u \in L(G)$ where $G$ is a generic subset of $\mathcal{O}$. By $i i), \operatorname{dim} P(\mathcal{C} ; u) \frac{u}{\overline{u_{0}}} \chi(\mathcal{C} ; \mathbf{T})$.

If $\mathcal{C}$ is stretched, by (2) and $i$ ), for all $u \in L(G)$ where $G$ is a generic subset of $\mathcal{O}$, $\operatorname{dim} P(\mathcal{C} ; u)$ is the generic dimension of $H\left(u^{\left(\cap_{\mathcal{C}}\left(A \cup T^{c}\right)\right)}\right)$ which is $d^{*}=\#\left(\cap_{\mathcal{C}} A\right)-1$. (Recall $\cup_{\mathcal{C}} T=\mathrm{T}$.) By $\left.i i\right), \operatorname{dim} P(\mathcal{C} ; u) \frac{u}{\bar{u}_{0}} d^{*}$. On the other hand, for all $u \in \mathcal{U}_{0}$, the induced utility plane of each bisupport of $\mathcal{C}$ contains $H\left(u^{\left(\cap_{\mathcal{C}} A\right)}\right)$, and hence so does $P(\mathcal{C} ; u)$. However, the generic dimension of $H\left(u^{\left(\cap_{\mathcal{c}} A\right)}\right)$ is $\#\left(\cap_{\mathcal{C}} A\right)-1=d^{*}$. Therefore, we conclude that $P(\mathcal{C} ; u) \frac{u}{\bar{u}_{0}} H\left(u^{(\cap A)}\right)$.

We complete the proof by constructing $L$ as described above. For each $t \in \mathbf{T}$, let $e^{t} \in \mathbb{R}^{\mathbf{T}}$ be a unit vector whose coordinate value is 1 for $t$ and 0 elsewhere. Let $\mathbf{e} \in \mathbb{R}^{\mathbf{T} \times \mathbf{T}}$ be such that $\mathbf{e}^{t}=e^{t}$ for all $t \in \mathbf{T}$. Let $\Omega \subset \mathbb{R}^{\mathbf{T} \times \mathbf{T}}$ be a small neighborhood of e such that, for all $\omega \in \Omega,\left\{\omega^{t}: t \in \mathbf{T}\right\}$ form a basis for $\mathbb{R}^{\mathbf{T}}$. Define $L: \mathcal{U}_{0} \times \Omega \rightarrow \mathcal{U}_{0}$ by

$$
L(u, \omega)=\omega^{-1} \cdot u
$$

We need to show that $L$ satisfies the properties $i$ ) and $i$ ) assumed above. Since $L(\cdot, \omega): \mathcal{U}_{0} \rightarrow \mathcal{U}_{0}$ is simply a nonsingular change of coordinate system expressed by $\omega^{-1}$, which itself is a smooth function of $\omega, L(\cdot, \cdot)$ is a surjective submersion. Moreover, $L(\cdot, \cdot)$ is a matrix of rational functions so that the image of a semi-algebraic set is semi-algebraic. Therefore, by Lemma 3.3, property $i i$ ) is proved. To show property $i$ ), consider a bisupport $(A, T) \in \mathcal{C}$ and $\left(A \cup T^{c}, \mathbf{T}\right)$. For $(u, \omega) \in \mathcal{U}_{0} \times \Omega$, the induced utility plane of $\left(A \cup T^{c}, \mathbf{T}\right)$ is $H\left(u^{A} \cup \omega^{T^{c}}\right)$ where $u^{A}=\left\{u^{a}: a \in A\right\}$ and $\omega^{T^{c}}=\left\{\omega^{t}: t \in T^{c}\right\}$. The transformation of this plane by $\omega^{-1}$ coincides with

$$
H\left(\omega^{-1}\left(u^{A} \cup \omega^{T^{c}}\right)\right)=H\left(\omega^{-1}\left(u^{A}\right) \cup\left\{e^{t}: t \notin T\right\}\right)=H\left(L(u, \omega)^{A} \cup\left\{e^{t}: t \notin T\right\}\right)
$$

Since $a_{0} \in A$ and $L(u, \omega) \in \mathcal{U}_{0}, H\left(L(u, \omega)^{A}\right)$ is a linear subspace. Hence, the affine subspace on the right hand side of $(4 . i)$ is $\phi_{T}^{-1} \phi_{T}\left(H\left(L(u, \omega)^{A}\right)\right)$ which is the induced utility 
plane of $(A, T)$ for $L(u, \omega)$. Since this is true for every bisupport of $\mathcal{C}^{c a}, P(\mathcal{C} ; L(u, \omega))$ is $P\left(\mathcal{C}^{c a} ;(u, \omega)\right)$ transformed by $\omega^{-1}$. Since $\omega^{-1}$ is a nonsingular linear transformation, $\operatorname{dim} P(\mathcal{C} ; L(u, \omega))=\operatorname{dim} P\left(\mathcal{C}^{c a} ;(u, \omega)\right)$.

\subsection{Non-annexed configurations}

In this subsection, we investigate the induced utility planes of non-annexed configurations. Earlier, we argued that if a configuration $\mathcal{C}$ on $\{\mathbf{A}, \mathbf{T}\}$ is induced by an equilibrium of $(\mathbf{A}, \mathbf{T}, \pi, u, v), \cup_{\mathcal{c}} T=\mathbf{T}$. However, we need to investigate subconfigurations for a more complete characterization of the induced utility plane of a non-annexed configuration, and a subconfiguration may not have this property. If $\left(\cup_{c} T\right)^{c} \neq \emptyset$, we may disregard types in $\left(\cup_{\mathcal{c}} T\right)^{c}$ because they play no role according to the configuration $\mathcal{C}$, that is, we may take $\cup_{\mathcal{C}} T$ as the underlying set of Sender types. This change of underlying Sender types reduces the $\chi$-value $\left(\chi\left(\mathcal{C} ; \cup_{\mathcal{C}} T\right)\right.$ as opposed to $\left.\chi(\mathcal{C} ; \mathbf{T})\right)$ and the dimension of the induced utility plane of $\mathcal{C}$ by $\#\left(\cup_{\mathcal{C}} T\right)^{c}$, which is natural because we are eliminating superfluous types. Now we state the result.

Proposition 4.8: Given a game form $(\mathbf{A}, \mathbf{T}, \pi)$, let $\mathcal{C}$ be a just and non-annexed configuration on $\{\mathbf{A}, \mathbf{T}\}$. Suppose $\mathcal{C}$ is trim. Let $\mathcal{U}=\mathbb{R}^{\mathbf{A} \times \mathbf{T}}$.

(a) If $\chi\left(\mathcal{C}^{\prime} ; \cup_{\mathcal{C}^{\prime}} T\right) \geq-1$ for all proper subconfiguration $\mathcal{C}^{\prime}$, then $\operatorname{dim} P(\mathcal{C} ; u) \frac{u}{\bar{u}} \chi(\mathcal{C} ; \mathbf{T})$.

(b) If $\chi\left(\mathcal{C}^{\prime} ; \cup_{\mathcal{C}^{\prime}} T\right)<-1$ for a proper subconfiguration $\mathcal{C}^{\prime}$, then $P(\mathcal{C} ; u) \frac{u}{\bar{u}} \emptyset$.

The main idea of the proof is as follows. We construct a hypothetical configuration with an artificial action $a_{0}$ common to all bisupports in a game form with one more type. The hypothetical configuration is annexed by construction, so we have information about the induced utility planes of this configuration by Proposition 4.8. From this, we derive information about the induced utility planes of the original configuration.

Formally, let $\mathbf{T}^{E}=\mathbf{T} \cup\left\{t_{0}\right\}$ and $\mathbf{A}^{E}=\mathbf{A} \cup\left\{a_{0}\right\}$ for some $a_{0} \notin \mathbf{A}$ and $t_{0} \notin \mathbf{T}$, and define a new configuration $\mathcal{C}^{E}$ on $\left\{\mathbf{T}^{E}, \mathbf{A}^{E}\right\}$, the annexation of $\mathcal{C}$, as

$$
\mathcal{C}^{E}=\left\{\left(A \cup\left\{a_{0}\right\}, T \cup\left\{t_{0}\right\}\right):(A, T) \in \mathcal{C}\right\}
$$

For $\mathcal{C}^{E}$, the space of expectation vectors is $\mathbb{R}^{\mathbf{T}^{E}}$. Since $\mathcal{C}^{E}$ is annexed by construction, by 
Remark 4.3, we may apply Proposition 4.2 with respect to

$$
\mathcal{U}_{0}^{E}=\left\{\mathrm{u} \in \mathbb{R}^{\mathbf{A}^{E} \times \mathbf{T}^{E}}: \mathbf{u}^{a_{0}}=0\right\} \approx \mathbb{R}^{\mathbf{A} \times \mathbf{T}^{E}}
$$

Let $\mathbb{R}^{\mathbf{T}} \subset \mathbb{R}^{\mathbf{T}^{E}}$ denote the hyperplane $\left\{x \in \mathbb{R}^{\mathbf{T}^{E}}: x_{t_{0}}=0\right\}$ and let $\widetilde{\mathbb{R}}^{\mathbf{T}}=\left\{x \in \mathbb{R}^{\mathbf{T}^{E}}\right.$ : $\left.x_{t_{0}}=1\right\}$, a translation of $\mathbb{R}^{\mathbf{T}}$ by $e^{t_{0}}$. We take $\widetilde{\mathbb{R}}^{\mathbf{T}}$ as the space of expectation vectors for $\mathcal{C}$, so that the space of utilities for $\mathcal{C}$ is $\mathcal{U}=\left(\widetilde{\mathbb{R}}^{\mathbf{T}}\right)^{\mathbf{A}}$. Then, the induced utility planes of bisupports in $\mathcal{C}^{E}$ are linear subspaces of $\mathbb{R}^{T^{E}}$ while those of bisupports in $\mathcal{C}$ are affine subspaces of $\widetilde{\mathbb{R}}^{\mathbf{T}}$.

Prior to a formal proof of Proposition 4.8, some preliminary results need to be established. Geometrically, there is one to one correspondence between affine subspaces of $\widetilde{\mathbb{R}}^{\mathrm{T}}$ and linear subspaces of $\mathbb{R}^{\mathrm{T}^{E}}$ which do not fall within $\mathbb{R}^{\mathrm{T}}$. Lemma 4.9 is on geometric implication of this correspondence which will be used to establish relationship between the induced utility plane of $\mathcal{C}$ and that of $\mathcal{C}^{E}$. Lemmas $4.10-4.12$ are on the properties of $\mathcal{C}^{E}$ implied by properties of $\mathcal{C}$.

Lemma 4.9 : Let $Z^{1}, \ldots, Z^{J}$ be linear subspaces of $\mathbb{R}^{\mathbf{T}^{E}}$.

(a) $\cap_{j=1}^{J}\left(Z^{j} \cap \widetilde{\mathbb{R}}^{\mathbf{T}}\right)=\left(\cap_{j=1}^{J} Z^{j}\right) \cap \widetilde{\mathbb{R}}^{\mathbf{T}}$.

(b) If $\cap_{j=1}^{J} Z^{j} \subset \mathbb{R}^{\mathrm{T}}$, then $\cap_{j=1}^{J}\left(Z^{j} \cap \widetilde{\mathbb{R}}^{\mathrm{T}}\right)=\emptyset$.

(c) If $\cap_{j=1}^{J} Z^{j} \not \subset \mathbb{R}^{\mathbf{T}}$, then $\operatorname{dim}\left(\cap_{j=1}^{J}\left(Z^{j} \cap \tilde{\mathbb{R}}^{\mathbf{T}}\right)\right)=\operatorname{dim}\left(\cap_{j=1}^{J} Z^{j}\right)-1$.

Proof : Part (a) is clear from repeated application of commutativity of intersection. Parts (b) and (c) are immediate consequences of (a).

Lemma 4.10: Let $\mathcal{C}$ be a configuration on $\{\mathbf{A}, \mathbf{T}\}$.

(i) $\chi\left(\mathcal{C}^{E} ; \mathbf{T}^{E}\right)=\chi(\mathcal{C} ; \mathbf{T})+1$

(ii) $\chi\left(\mathcal{C} ; \cup_{\mathcal{c}} T\right)=\chi(\mathcal{C} ; \mathbf{T})-\#\left(\cap_{\mathcal{C}} T^{c}\right)$

Proof: (i) $\chi\left(\mathcal{C}^{E} ; \mathbf{T}^{E}\right)=\sum_{\mathcal{c}}\left(\# A-{ }^{\#} T\right)+(\# \mathbf{T}+1)-\# \mathcal{C}=\chi(\mathcal{C} ; \mathbf{T})+1$.

(ii) $\chi\left(\mathcal{C} ; \cup_{\mathcal{C}} T\right)=\sum_{\mathcal{C}}\left(\#_{A}-\#_{T}\right)+\#\left(\cup_{\mathcal{C}} T\right)-\# \mathcal{C}=\chi(\mathcal{C} ; \mathbf{T})-\#\left(\cap_{\mathcal{C}} T^{c}\right)$.

Lemma 4.11: Let $\mathcal{C}$ be a configuration as described in Proposition 4.8 and $\mathcal{C}^{E}$ be the annexation. Then,

(i) $\mathcal{C}^{E}$ is just and every proper subconfiguration of $\mathcal{C}^{E}$ is unstretched, and 
(ii) $\mathcal{C}^{E}$ is trim if and only if $\chi(\mathcal{C} ; \mathbf{T}) \geq-1$.

Proof: Since $\mathcal{C}$ is just, $\#_{A} \leq \#^{T}$ for all $(A, T) \in \mathcal{C}$ so that $\#^{\#}\left(A \cup\left\{a_{0}\right\}\right) \leq \#\left(T \cup\left\{t_{0}\right\}\right)$. Clearly, the union of all type components of $\mathcal{C}^{E}$ is $\mathbf{T}^{E}$. Hence, $\mathcal{C}^{E}$ is just.

Clearly, a subconfiguration of $\mathcal{C}^{E}$ is the annexation of a subconfiguration of $\mathcal{C}$. Let $\mathcal{C}^{\prime E}$ denote the annexation of $\mathcal{C}^{\prime} \subset \mathcal{C}$. Then, $\mathcal{C}^{\prime E}$ has exactly one more common action, $a_{0}$, than $\mathcal{C}^{\prime}$. On the other hand, $\chi\left(\mathcal{C}^{E} ; \mathbf{T}^{E}\right)=\chi\left(\mathcal{C}^{\prime} ; \mathbf{T}\right)+1$ by Lemma $4.10(i)$. Therefore, from the definition of "unstretched" (Definition 2.8), $\mathcal{C}^{E}$ is unstretched if $\chi\left(\mathcal{C}^{\prime} ; \mathbf{T}\right) \geq-1$.

If $\mathcal{C}^{\prime E}$ is a proper subconfiguration, $\mathcal{C}^{\prime}$ is also a proper subconfiguration of $\mathcal{C}$ so that $\chi\left(\mathcal{C}^{\prime} ; \cap_{\mathcal{C}} T\right) \geq-1$. By Lemma $4.10(i i), \chi\left(\mathcal{C}^{\prime} ; T\right)=\chi\left(\mathcal{C}^{\prime} ; \cap_{\mathcal{C}} T\right)+{ }^{\#}\left(\cap_{\mathcal{C}^{\prime}} T^{c}\right) \geq-1$ and $\mathcal{C}^{\prime E}$ is unstretched. So, $(i)$ is proved.

Next consider $\mathcal{C}^{E}$ itself. Clearly from construction, $\left\{a_{0}\right\}$ is the only common action of all bisupports of $\mathcal{C}^{E}$ and the union of all type components of $\mathcal{C}^{E}$ is $\mathbf{T}^{E}$. Hence, from Definition $2.8, \mathcal{C}^{E}$ is unstretched if and only if $\chi(\mathcal{C}) \geq-1$. This proves $(i i)$.

Lemma 4.12: Let $\mathcal{C}$ be a configuration as described in Proposition 4.8 and $\mathcal{C}^{E}$ be the annexation. Then, $\mathcal{C}^{E} \cup\left\{\left(\left\{a_{0}\right\}, \mathbf{T}\right)\right\}$ is trim if $\chi(\mathcal{C} ; \mathbf{T}) \geq-1$.

Proof: Subconfigurations of $\mathcal{C}^{E}$ are unstretched by Lemma 4.11. Now, consider a configuration of the form $\mathcal{C}^{\prime E} \cup\left\{\left(\left\{a_{0}\right\}, \mathbf{T}\right)\right\}$ where $\mathcal{C}^{\prime E}$ is the annexation of $\mathcal{C}^{\prime} \subset \mathcal{C}$. Clearly, $a_{0}$ is the only common action to this configuration. Moreover, from construction of $\mathcal{C}^{E}$, the union of all type components of this configuration is $\mathbf{T}^{E}$. So, to show that this configuration is unstretched, we need to show $\chi\left(\mathcal{C}^{E} \cup\left\{\left(\left\{a_{0}\right\}, \mathbf{T}\right)\right\} ; \mathbf{T}^{E}\right)+1 \geq 1$.

This is immediate for $\mathcal{C}^{\prime}=\mathcal{C}$ from the hypothesis that $\chi(\mathcal{C} ; T) \geq-1$. To examine $\mathcal{C}^{\prime} \neq \mathcal{C}$, observe that, by Lemma $4.10(i)$ and Lemma $4.4(\mathrm{a})$,

$$
\chi\left(\mathcal{C}^{\prime E} \cup\left\{\left(\left\{a_{0}\right\}, \mathbf{T}\right)\right\} ; \mathbf{T}^{E}\right)=\left(\chi\left(\mathcal{C}^{\prime} ; \mathbf{T}\right)+1\right)+\# \mathbf{T}-(\# \mathbf{T}+1)=\chi\left(\mathcal{C}^{\prime} ; \mathbf{T}\right)+2
$$

Since $\chi\left(\mathcal{C}^{\prime} ; \cup_{\mathcal{C}} T\right) \geq-1$ by hypothesis, $\chi\left(\mathcal{C}^{\prime} ; \mathbf{T}\right)=\#\left(\cap_{\mathcal{C}^{\prime}} T^{c}\right)+\chi\left(\mathcal{C}^{\prime} ; \cup_{\mathcal{C}} T\right) \geq-1$ by Lemma 4.10 (ii). So, from (4.j), $\chi\left(\mathcal{C}^{\prime E} \cup\left\{\left(\left\{a_{0}\right\}, T\right)\right\}\right)+1 \geq 1$ as pursued.

With these supplimentary results, we first prove part (a) of Proposition 4.8.

Proof of Proposition 4.8 (a): Let $\mathcal{C}$ be a configuration as described in Proposition 4.8 (a) and $\mathcal{C}^{E}, \mathcal{U}, \mathcal{U}_{0}^{E}$ and $\widetilde{\mathbb{R}}^{\mathrm{T}}$ be as explained above. First we will prove the result 
using a hypothetical function $\Psi$ as described below, after which we complete the proof by constructing $\Psi$.

Suppose there is a mapping $\Psi: \mathcal{O} \rightarrow \mathcal{U}$, where $\mathcal{O}$ is an open subset of $\mathcal{U}_{0}^{E}$, such that

i) $P\left(\left\{\left(A \cup\left\{a_{0}\right\}, T \cup\left\{t_{0}\right\}\right)\right\} ; \mathrm{u}\right) \cap \widetilde{\mathbb{R}}^{\mathrm{T}}=P(\{(A, T)\} ; \Psi(\mathrm{u}))$ for all $\mathrm{u} \in \mathcal{O}$, and

ii) $\Psi(G)$ is a generic subset of $\mathcal{U}$ if $G$ is a generic subset of $\mathcal{O}$.

We may express the induced utility plane of a bisupport as that of the configuration consisting of that bisupport. So, $P(\mathcal{C} ; \Psi(\mathrm{u}))$ is the intersection of $P\left(\left\{\left(A \cup\left\{a_{0}\right\}, T \cup\left\{t_{0}\right\}\right)\right\} ; \mathrm{u}\right) \cap \widetilde{\mathbb{R}}^{\mathbf{T}}$ while $P\left(\mathcal{C}^{E} ; \mathbf{u}\right)$ is the intersection of $P\left(\left\{\left(A \cup\left\{a_{0}\right\}, T \cup\left\{t_{0}\right\}\right)\right\} ; \mathbf{u}\right)$ over all bisupports. In light of Lemma 4.9 (where $Z^{j}$ corresponds to a certain $P\left(\left\{\left(A \cup\left\{a_{0}\right\}, T \cup\left\{t_{0}\right\}\right)\right\} ; \mathrm{u}\right)$ ), $\operatorname{dim} P(\mathcal{C} ; \Psi(\mathbf{u}))$ depends on whether $P\left(\mathcal{C}^{E} ; \mathbf{u}\right) \subset \mathbb{R}^{\mathbf{T}}$.

Consider a configuration $\mathcal{C}$ described in Proposition 4.8 (a) with a negative $\chi$-value. If $\chi(\mathcal{C} ; \mathbf{T})=-1, \mathcal{C}^{E}$ is trim by Lemma 4.11 , but it is taut with $\chi\left(\mathcal{C}^{E} ; \mathbf{T}^{E}\right)=0$ so that $P\left(\mathcal{C}^{E} ; \mathbf{u}\right) \frac{\mathrm{u}}{{\overline{u_{0}^{E}}}_{y^{E}}}\left\{\mathbf{u}^{a_{0}}\right\}$ by Proposition $4.2(\mathrm{a})$. If $\chi(\mathcal{C})<-1, \mathcal{C}^{E}$ is subject to Proposition 4.2 (b) by Lemma 4.11. So, $P\left(\mathcal{C}^{E} ; \mathbf{u}\right) \frac{\underline{u}}{\overline{u_{0}^{E}}}\left\{\mathbf{u}^{a_{0}}\right\}$. In either case, $P\left(\mathcal{C}^{E} ; \mathbf{u}\right)=\left\{\mathbf{u}^{a_{0}}\right\}=\{0\} \subset \mathbb{R}^{\mathbf{T}}$ for all $u$ in a generic set of utilities $G \subset \mathcal{O}$. Therefore, $P(\mathcal{C} ; u)=\emptyset$ for all $u \in \Psi(G)$ by Lemma 4.9 (a). Since negative dimensions denote empty set, by property $i i$ ) of $\Psi$, we have proved $\operatorname{dim} P(\mathcal{C} ; u) \frac{u}{\bar{u}} \chi(\mathcal{C} ; \mathbf{T})$ for configurations with negative $\chi$-values.

For configurations with nonnegative $\chi$-values, we will check whether $P\left(\mathcal{C}^{E} ; \mathbf{u}\right) \subset$ $\mathbb{R}^{\mathbf{T}}$ by comparing $\operatorname{dim} P\left(\mathcal{C}^{E} ; \mathbf{u}\right)$ and $\operatorname{dim}\left(P\left(\mathcal{C}^{E} ; \mathbf{u}\right) \cap \mathbb{R}^{\mathbf{T}}\right)$; if they $\operatorname{differ} P\left(\mathcal{C}^{E} ; \mathbf{u}\right) \not \subset \mathbb{R}^{\mathbf{T}}$. However, since $\mathbb{R}^{\mathbf{T}}$ is the induced utility plane of $\left(\left\{a_{0}\right\}, \mathbf{T}\right), P\left(\mathcal{C}^{E} ; \mathbf{u}\right) \cap \mathbb{R}^{\mathbf{T}}$ is the induced utility plane of $\mathcal{C}^{E} \cup\left\{\left(\left\{a_{0}\right\}, \mathbf{T}\right)\right\}$.

If $\chi(\mathcal{C}) \geq 0, \mathcal{C}^{E}$ and $\mathcal{C}^{E} \cup\left\{\left(\left\{a_{0}\right\}, T\right)\right\}$ are trim by Lemmas 4.11 and 4.12. By Proposition 4.2 applied to both, there exists a generic set of utilities $G \subset \mathcal{O}$ such that $\operatorname{dim} P\left(\mathcal{C}^{E} ; \mathbf{u}\right)=\chi\left(\mathcal{C}^{E} ; \mathbf{T}^{E}\right)$ and $\operatorname{dim}\left(P\left(\mathcal{C}^{E} ; \mathbf{u}\right) \cap \mathbb{R}^{\mathbf{T}}\right)=\chi\left(\mathcal{C}^{E} \cup\left\{\left(\left\{a_{0}\right\}, \mathbf{T}\right)\right\} ; \mathbf{T}^{E}\right)$ for all $\mathbf{u} \in G$. By Lemma 1.2 $(i), \chi\left(\mathcal{C}^{E} \cup\left\{\left(\left\{a_{0}\right\}, \mathbf{T}\right)\right\} ; \mathbf{T}^{E}\right)=\chi\left(\mathcal{C}^{E} ; \mathbf{T}^{E}\right)+\# \mathbf{T}-\left({ }^{\#} \mathbf{T}+1\right)=$ $\chi\left(\mathcal{C}^{E} ; \mathbf{T}^{E}\right)-1$. This means that $P\left(\mathcal{C}^{E} ; \mathrm{u}\right) \not \subset \mathbb{R}^{\mathbf{T}}$ for all $\mathrm{u} \in G$. By Lemmas 4.9 (c) and $4.10(i), \operatorname{dim} P(\mathcal{C} ; u)=\chi\left(\mathcal{C}^{E} ; \mathbf{T}^{E}\right)-1=\chi(\mathcal{C} ; \mathbf{T})$ for all $u$ in $\Psi(G)$ which is a generic subset of $\mathcal{U}$ by property $i$ ) of $\Psi$. This completes the proof of Proposition 4.8 (a) if there exists $\Psi$ as described above. 
We complete the proof by constructing $\Psi$. Let $\psi: \mathbb{R}^{\mathbf{T}^{E}} \backslash \mathbb{R}^{\mathbf{T}} \rightarrow \widetilde{\mathbb{R}}^{\mathbf{T}}$ be the projection through the origin, i.e,

$$
\psi(x)=\frac{x}{x_{t_{0}}}, \quad x \in \mathbb{R}^{\mathbf{T}^{E}} \backslash \mathbb{R}^{\mathbf{T}}
$$

Being a projection through the origin, $\psi$ is a surjective submersion. Take an open subset of $\mathcal{U}_{0}^{E}$ as $\tilde{\mathcal{U}}_{0}^{E}=\left\{\mathbf{u} \in \mathcal{U}_{0}^{E}: \mathbf{u}^{a} \in \mathbb{R}^{\mathbf{T}^{E}} \backslash \mathbb{R}^{\mathbf{T}}, \forall a \in \mathbf{A}\right\}$, and let $\Psi: \tilde{\mathcal{U}}_{0}^{E} \rightarrow \mathcal{U}$ be the projection of utility vector of each action by $\psi$, i.e,

$$
\boldsymbol{\Psi}(\mathrm{u})=\left(\psi\left(\mathbf{u}^{a}\right)\right)_{a \in \mathbf{A}}
$$

We need to show properties $i$ ) and $i i)$. Since $\psi$ is a surjective submersion, so is $\boldsymbol{\Psi}$. Moreover, since $\psi$ (hence $\Psi$ ) is a rational function coordinatewise, $\Psi$ maps a semi-algebraic set to a semi-algebraic set. Hence, by Lemma 3.3, property $i i)$ is proved. To show property i), consider $P\left(\left\{\left(A \cup\left\{a_{0}\right\}, T \cup\left\{t_{0}\right\}\right)\right\} ; \mathrm{u}\right)$ and $P(\{(A, T)\} ; \Psi(\mathrm{u}))$ for $\mathrm{u} \in \tilde{\mathcal{U}}_{0}^{E}$. The former is $\operatorname{span}\left\{\mathbf{u}^{a}: a \in A\right\}$ extended along the utility directions of $\left(T^{E}\right)^{c}$ while the latter is $H\left(\left\{\psi\left(\mathbf{u}^{a}\right): a \in A\right\}\right)$ extended along the utility directions of $T^{c}$. Since $\mathbf{u} \in \tilde{\mathcal{U}}_{0}^{E}$,

$$
\operatorname{span}\left\{\mathbf{u}^{a}: a \in A\right\} \cap \widetilde{\mathbb{R}}^{\mathbf{T}}=H\left(\left\{\psi\left(\mathbf{u}^{a}\right): a \in A\right\}\right)
$$

Furthermore, $\left(T^{E}\right)^{c}=T^{c} \subset \mathbf{T}$, which means that the utility directions for extension are common to both induced utility planes and are contained in $\mathbb{R}^{\mathrm{T}}$. Therefore, every point in $P\left(\left\{\left(A \cup\left\{a_{0}\right\}, T \cup\left\{t_{0}\right\}\right)\right\} ; \mathbf{u}\right) \cap \widetilde{\mathbb{R}}^{\mathbf{T}}$ is either in or extended from $\operatorname{span}\left\{\mathbf{u}^{a}: a \in A\right\} \cap \widetilde{\mathbb{R}}^{\mathbf{T}}$. Hence, we conclude $P\left(\left\{\left(A \cup\left\{a_{0}\right\}, T \cup\left\{t_{0}\right\}\right)\right\} ; \mathrm{u}\right) \cap \widetilde{\mathbb{R}}^{\mathbf{T}}=P(\{(A, T)\} ; \Psi(\mathrm{u}))$.

To prove part (b) of Proposition 4.8, we need a Lemma on how properties of a configuration change when the underlying set of Sender types varies. Given a configuration $\mathcal{C}$ on $\{\mathbf{A}, \mathbf{T}\}$, notation $\left.\mathcal{C}\right|_{\mathbf{T}^{\prime}}$ is used to denote the same configuration with underlying set of Sender types $\mathbf{T}^{\prime} \subset \mathbf{T}$.

Lemma 4.13 : Let $\mathcal{C}$ be a configuration on $\{\mathbf{A}, \mathbf{T}\}$ and let $\emptyset \neq \mathcal{C}^{\prime \prime} \subset \mathcal{C}^{\prime} \subset \mathcal{C}$. Then, $\left.\mathcal{C}^{\prime \prime}\right|_{\left(\cup_{\mathcal{C}^{\prime}} T\right)}$ is unstretched if and only if one of the following alternatives holds:

i) $\mathcal{C}^{\prime \prime}$ is unstretched, or

ii) $\mathcal{C}^{\prime \prime}$ is stretched, $\cap_{\mathcal{C}^{\prime \prime}} A=\emptyset$ and $\cup_{\mathcal{C}^{\prime \prime}} T=\cup_{\mathcal{C}^{\prime}} T$. 
Proof : From formulae (2.d) and (2.e) in Definition 2.9,

$$
\begin{aligned}
& \left(\text { LHS of }(2 . e) \text { for }\left.\mathcal{C}^{\prime \prime}\right|_{\left(\cup_{\mathcal{c}^{\prime}} T\right)}\right)=\left(\text { LHS of }(2 . e) \text { for } \mathcal{C}^{\prime \prime}\right)-{ }^{\#}\left(\cup_{\mathcal{C}} T\right)^{c} \\
& \chi\left(\left.\mathcal{C}^{\prime \prime}\right|_{\left(\cup_{\mathcal{C}^{\prime}} T\right)} ; \cup_{\mathcal{C}^{\prime}} T\right)=\chi\left(\mathcal{C}^{\prime \prime} ; T\right)-{ }^{\#}\left(\cup_{\mathcal{C}} T\right)^{c}
\end{aligned}
$$

where LHS means left hand side. Below, RHS means right hand side.

If $\mathcal{C}^{\prime \prime}$ is unstretched, LHS of (2.e) $\leq$ RHS of (2.e) for $\mathcal{C}^{\prime \prime}$. Hence, by (4.n) and (4.o), the relation holds for $\left.\mathcal{C}^{\prime \prime}\right|_{\left(\cup_{\mathcal{c}^{\prime}} T\right)}$ also. Next, if $\mathcal{C}^{\prime \prime}$ is stretched, $\cap_{\mathcal{C}^{\prime \prime}} A=\emptyset$ and $\cup_{\mathcal{C}^{\prime \prime}} T=\cup_{\mathcal{C}^{\prime}} T$, then LHS of (2.e) is 0 for $\left.\mathcal{C}^{\prime \prime}\right|_{\left(U_{\mathcal{c}^{\prime}} T\right)}$ so that it is trivially unstretched.

The remaining possibility is $\mathcal{C}^{\prime \prime}$ is stretched but either $\cap_{\mathfrak{C}^{\prime \prime}} A \neq \emptyset$ or $\cup_{\mathcal{C}^{\prime \prime}} T \neq \cup_{\mathcal{C}^{\prime}} T$. Since $\mathcal{C}^{\prime \prime}$ is stretched, LHS of (2.e) > RHS of (2.e) for $\mathcal{C}^{\prime \prime}$. In this case, however, LHS of (2.e) is positive for $\left.\mathcal{C}^{\prime \prime}\right|_{\left(\cup_{\mathcal{C}^{\prime}} T\right)}$. Hence, by (4.n) and (4.o), LHS of (2.e) > RHS of (2.e) for $\left.\mathcal{C}^{\prime \prime}\right|_{\left(\cup_{\mathcal{C}}, T\right)}$. This completes the proof.

Proof of Proposition 4.8 (b) : Consider a configuration $\mathcal{C}$ as decribed in Proposition 4.8 (b). Then, we can find a subconfiguration $\mathcal{C}^{\prime}$ such that $\chi\left(\mathcal{C}^{\prime} ; \cup_{\mathcal{C}^{\prime}} T\right)<-1$ which is minimal in the sense that no proper subconfiguration of $\mathcal{C}^{\prime}$ has this property. Consider $\left.\mathcal{C}^{\prime}\right|_{\left(\cup_{\mathcal{C}^{\prime}} T\right)}$.

By Lemma 4.13, this configuration is trim and by Proposition $4.8(\mathrm{a}), P\left(\left.\mathcal{C}^{\prime}\right|_{\left(U_{\mathcal{C}^{\prime}} T\right)} ; u^{\prime}\right) \frac{u^{\prime}}{\overline{u^{\prime}}} \emptyset$ where $\mathcal{U}^{\prime}=\mathbb{R}^{\mathbf{A} \times\left(\cup_{\mathcal{c}^{\prime}} T\right)}$ is the utility space for $\left.\mathcal{C}^{\prime}\right|_{\left(\cup_{\mathcal{c}^{\prime}} T\right)}$. Notice here that $P\left(\left.\mathcal{C}^{\prime}\right|_{\left(\cup_{\mathcal{c}}, T\right)} ; u^{\prime}\right) \subset$ $\mathbb{R}^{\left(\cup_{\mathcal{c}^{\prime}}, T\right)}$ for $u^{\prime} \in \mathcal{U}^{\prime}$ while $P\left(\mathcal{C}^{\prime} ; u\right) \subset \mathbb{R}^{T}$ for $u \in \mathcal{U}$. Moreover, if $u^{\prime}$ is $u$ restricted to $\cup_{\mathcal{C}^{\prime}} T$, then $P\left(\left.\mathcal{C}^{\prime}\right|_{\left(\cup_{\mathcal{C}^{\prime}}, T\right)} ; u^{\prime}\right)=\phi_{\left(\cup_{\mathcal{C}^{\prime}} T\right)}\left(P\left(\mathcal{C}^{\prime} ; u\right)\right)$ where $\phi_{\left(\cup_{\mathcal{C}^{\prime}} T\right)}$ is the natural projection of $\mathbb{R}^{\mathbf{T}}$ onto $\mathbb{R}^{\left(\cup_{\mathcal{C}^{\prime}} T\right)}$. Hence, $P\left(\mathcal{C}^{\prime} ; u\right)=\emptyset$ if $P\left(\left.\mathcal{C}^{\prime}\right|_{\left(\cup_{\mathcal{C}}, T\right)} ; u^{\prime}\right)=\emptyset$. Therefore, $P\left(\mathcal{C}^{\prime} ; u\right) \frac{u}{\bar{u}} \emptyset$ which implies $P(\mathcal{C} ; u) \frac{u}{\bar{u}} \emptyset$.

\subsection{Implication}

Recall Remark 2.8 which justified that we only need to consider just configurations. Given a game form $(\mathbf{A}, \mathbf{T}, \pi)$, let $\mathcal{C}$ be a just configuration on $\{\mathbf{A}, \mathbf{T}\}$ and let $\mathcal{U}=\mathbb{R}^{\mathbf{A} \times \mathbf{T}}$. First, suppose $\mathcal{C}$ is trim. Then, the generic dimension of the induced utility plane of $\mathcal{C}$ is $\chi(\mathcal{C} ; \mathrm{T})$ by Propositions 4.2 (a) or $4.8(\mathrm{a})$ unless $\mathcal{C}$ is non-annexed and $\chi\left(\mathcal{C}^{\prime} ; \cup_{\mathcal{C}}, T\right)<-1$ for a proper subconfiguration $\mathcal{C}^{\prime}$. In the latter case, $P(\mathcal{C} ; u) \frac{u}{\bar{u}} \emptyset$ by Proposition 4.8 (b). Recall that $P(\mathcal{C} ; u)$ contains the set of all expectation vectors that satisfy optimality condition (SO) with respect to $\mathcal{C}$. Hence, if a configuration $\mathcal{C}$ on $\{\mathbf{A}, \mathbf{T}\}$ is trim and $\chi(\mathcal{C} ; \mathbf{T})<0$, there is no expectation vector that satisfies (SO) with respect to $\mathcal{C}$. This establishes $(\mathrm{C} 2)$. 
Next, suppose $\mathcal{C}$ is not trim. Then, we can find a stretched subconfiguration $\mathcal{C}^{\prime}$ of $\mathcal{C}$ which is minimal in the sense that no proper subconfiguration of $\mathcal{C}^{\prime}$ is stretched. Again, consider $\left.\mathcal{C}^{\prime}\right|_{\left(\cup_{\mathcal{C}^{\prime}} T\right)}$. By Lemma 4.13, every proper subconfiguration of $\left.\mathcal{C}^{\prime}\right|_{\left(\cup_{\mathcal{C}^{\prime}} T\right)}$ is unstretched.

If $\cap_{\mathcal{C}^{\prime}} A=\emptyset,\left.\mathcal{C}^{\prime}\right|_{\left(\cup_{\mathcal{C}^{\prime}}, T\right)}$ is unstretched by Lemma $4.13\left(\right.$ take $\left.\mathcal{C}^{\prime \prime}=\mathcal{C}^{\prime}\right)$ so that it is trim. Hence, by Proposition 4.8 applied to $\left.\mathcal{C}^{\prime}\right|_{\left(\cup_{\mathcal{C}^{\prime}} T\right)}$, either $\operatorname{dim} P\left(\left.\mathcal{C}^{\prime}\right|_{\left(\cup_{\mathcal{C}^{\prime}} T\right)} ; u^{\prime}\right) \frac{u^{\prime}}{\overline{u^{\prime}}} \chi\left(\mathcal{C}^{\prime} ; \cup_{\mathcal{C}^{\prime}} T\right)$ or $P\left(\left.\mathcal{C}^{\prime}\right|_{\left(\cup_{\mathcal{C}^{\prime}} T\right)} ; u^{\prime}\right) \frac{u^{\prime}}{\overline{\mathcal{U}^{\prime}}} \emptyset$ where $\mathcal{U}^{\prime}=\mathbb{R}^{\mathbf{A} \times\left(\cup_{\mathcal{C}^{\prime}}, T\right)}$. However, because $\cap_{\mathcal{C}^{\prime}} A=\emptyset, \mathcal{C}^{\prime}$ being stretched implies $\chi\left(\mathcal{C}^{\prime} ; \cup_{\mathcal{C}^{\prime}} T\right)<-1$. Hence, $P\left(\mathcal{C}^{\prime} ; u^{\prime}\right) \frac{u^{\prime}}{\overline{\mathcal{u}^{\prime}}} \emptyset$ if $\cap_{\mathcal{C}^{\prime}} A=\emptyset$. By the same reasoning as used in the proof of Proposition $4.8(\mathrm{~b})$, we conclude $P(\mathcal{C} ; u) \frac{u}{\bar{u}} \emptyset$. So, there is no expectation vector that satisfies ( $\mathrm{SO}$ ) for generic utilities.

If $\cap_{\mathcal{C}^{\prime}} A \neq \emptyset,\left.\mathcal{C}^{\prime}\right|_{\left(\cup_{\mathcal{C}^{\prime}} T\right)}$ is stretched by Lemma 4.13. Hence, $P\left(\mathcal{C}^{\prime} ; u^{\prime}\right) \frac{u^{\prime}}{\bar{u}^{\prime}} H\left(u^{\left(\cap_{\mathcal{C}^{\prime}} A\right)}\right)$ by Proposition 4.2 (b). Consider an expectation vector $u^{*} \in H\left(u^{\left(\cap_{\mathfrak{c}^{\prime}} A\right)}\right)$ as a candidate equilibrium expected utility vector. Since $\#\left(\cap_{\mathcal{C}^{\prime}} A\right) \leq \# \mathbf{T}, H\left(u^{\left(\cap_{\mathcal{C}^{\prime}} A\right)}\right)$ has full dimension $\#\left(\cap_{\mathcal{C}^{\prime}} A\right)-1$ for generic $u$ so that there is a unique response mixed over $\cap_{\mathcal{C}^{\prime}} A$ that induces $u^{*}$. For each $(A, T) \in \mathcal{C}^{\prime}$, the induced utility plane of $(A, T)$ is of full dimension generically. Hence, for generic utilities, there is a unique response mixed over $A$ that induces $u^{*}$ which is, in fact, the unique response mixed over $\cap_{\mathcal{C}} A$. Therefore, for generic utilities, if an equilibrium determines the configuration $\mathcal{C}$, the responses to messages corresponding to bisupports in $\mathcal{C}^{\prime}$ should be identical. This means that, for generic utilities, no equilibrium in $\mathcal{C}$ could be in reduced form.

Conclusively, if $\mathcal{C}$ is not trim, in either case considered above, there is no reduced form equilibrium in $\mathcal{C}$ for generic utilities, which establishes (C1).

Consider a just configuration $\mathcal{C}$ on $\{\mathbf{A}, \mathbf{T}\}$ such that $\#_{\mathcal{C}}>\#_{\mathbf{T}}$. Since $\#_{A}<\#_{T}$ for all $(A, T) \in \mathcal{C}, \chi(\mathcal{C} ; T)<0$ is immediate from the definition of $\chi$-value. Therefore, independently of whether $\mathcal{C}$ is trim or not, the game $(\mathbf{A}, \mathbf{T}, \pi, u, v)$ has no reduced form equilibrium in $\mathcal{C}$ for generic $u$. Since this conclusion comes from the hypothesis that $\#$ C $>$ \#T, we proved Theorem 2.2 (a). 


\section{Receiver's Optimality and Integrated Results}

In the previous section, we eliminated certain configurations as being incompatible with the Sender's optimality condition (SO) for generic utilities. The configurations that survived this process are just configurations which are trim with non-negative $\chi$-values. In this section, we investigate the compatibility with the Receiver's optimality condition (RO) for these configurations and establish the main results of the paper.

Recall the second necessary condition (RO) for a configuration to have an equilibrium in it, namely, there is a talking strategy the posterior profile of which matches the configuration in the following sense: there is a one to one and onto mapping between the posterior profile and the bisupports of the configuration such that $i$ ) the support of each posterior belief is the type component of the matched bisupport and $i i)$ the actions in each bisupport are best responses to the matched posterior belief. We look into the utilities of the Receiver that satisfy this property. Posteriors are meaningful when they give nonnegative probabilities to all types. But, for expositional simplicity, the subsequent discussion is carried out in the affine extension of $\Delta(\mathbf{T})$, that is, in $H(\mathbf{T}) \equiv\left\{p \in \mathbb{R}^{\mathbf{T}}: \sum_{t \in \mathbf{T}} p_{t}=1\right\}$.

Given a game form $(\mathbf{A}, \mathbf{T}, \pi)$, let $\mathcal{C}$ be a just configuration on $\{\mathbf{A}, \mathbf{T}\}$. Suppose there is a talking strategy $\tau$ which satisfies (RO) with respect to $\mathcal{C}$ for a utility $v$ of the Receiver. Then, for each bisupport $(A, T), \tau$ generates a posterior which assigns zero probability to types not in $T$, and for which all actions in $A$ are indifferent to the Receiver. Formally, this posterior belongs to

$$
\Pi((A, T) ; v) \equiv\left\{p \in H(\mathbf{T}): p_{t}=0 \text { if } t \notin T \text { and } p \cdot v^{a}=p \cdot v^{b} \text { for all } a \text { and } b \text { in } A\right\}
$$

where $v^{a} \in \mathbb{R}^{\mathbf{T}}$ denotes the utility vector that action $a$ induces to the Receiver for all possible Sender types. Since this holds for every bisupport and the prior $\pi$ is expressed as a convex combination of posterior beliefs of all used messages, it follows that $\pi \in$ $\Pi(\mathcal{C} ; v)$ where $\Pi(\mathcal{C} ; v) \equiv H\left(\cup_{\mathcal{C}} \Pi((A, T) ; v)\right)$. In other words, a necessary condition for a configuration $\mathcal{C}$ to have a talking strategy satisfying (RO) for $v$ is

$$
\pi \in \Pi(\mathcal{C} ; v)
$$

Hence, given a game form and a just configuration $\mathcal{C}$, we look for the utilities of the Receiver with this property. The main findings are stated below. 
Proposition 5.1 : Given a game form $(\mathbf{A}, \mathbf{T}, \pi)$ where $\pi \in \Delta^{\circ}(T)$, let $\mathcal{C}$ be a just configuration on $\{\mathbf{A}, \mathbf{T}\}$.

(a) If $\mathcal{C}$ is trim and $\chi(\mathcal{C} ; \mathbf{T})>0$, there is a generic subset $F$ of $\mathcal{V}$ such that $\pi \notin \Pi(\mathcal{C} ; v)$ for all $v \in F$.

(b) If $\mathcal{C}$ is trim and $\chi(\mathcal{C})=0$, there is a generic subset $F$ of $\mathcal{V}$ such that, for all $v \in \mathcal{V}$, at most one talking strategy $\tau$ satisfies (RO) with respect to $\mathcal{C}$.

If $\Pi(\mathcal{C} ; v)=H(\mathbf{T})$, obviously $\pi \in \Pi(\mathcal{C} ; v)$. So, we first look into the dimension of $\Pi(\mathcal{C} ; v)$. Next Lemma is on dimensions of $\Pi((A, T) ; v)^{(12)}$ and $\Pi(\mathcal{C} ; v)$ for generic $v$.

Lemma 5.2: Suppose $\mathcal{C}$ is a just configuration on $\{\mathbf{A}, \mathbf{T}\}$. Then, there is a generic subset $F$ in $\mathcal{V}$ such that, for all $v \in F$,

i) $\operatorname{dim} \Pi((A, T) ; v)=\#_{T}-{ }^{\#} A$ for all $(A, T) \in \mathcal{C}$, and

ii) $\operatorname{dim} \Pi(\mathcal{C} ; v) \leq \# \mathbf{T}-1-\chi(\mathcal{C} ; \mathbf{T})$.

Proof: Consider a bisupport $(A, T) \in \mathcal{C}$. Let $A=\left\{a_{1}, \ldots, a_{k}\right\}$ where $k=\# A$. Since the utilities for types not in $T$ play no role in determining $\Pi((A, T) ; v)$, we consider utilities in $\left.\mathcal{V}\right|_{T} \equiv \mathbb{R}^{\mathbf{A} \times T}$. Clearly, $\Pi((A, T) ; v) \subset H(T)$ where $H(T)$ is the affine extension of $\Delta(T)$. For $\left.v \in \mathcal{V}\right|_{T}, \Pi((A, T) ; v)$ is the set of points $p \in H(T)$ that solves

$$
\left(\begin{array}{c}
v^{a_{1}}-v^{a_{2}} \\
v^{a_{1}}-v^{a_{3}} \\
\vdots \\
v^{a_{1}}-v^{a_{k}}
\end{array}\right) \cdot p=0
$$

where $v^{a_{i}}$ is a row vector. For generic $v$ in $\left.\mathcal{V}\right|_{T}$, variables in the matrix are independent of one another so that the matrix has full rank. Hence, for generic $v$ in $\left.\mathcal{V}\right|_{T}$, equation (5.b) has a solution with degrees of freedom $(\# T-1)-(k-1)$. (Notice that $\operatorname{dim} H(T)=\# T-1)$ Moreover, because the equation is linear, the solutions form an affine subspace. Therefore, $\Pi((A, T) ; v)$ is a (\#T-k)-dimensional affine subspace of $H(T)$ for utilities in a generic subset, say $F_{(A, T)}$ of $\left.\mathcal{V}\right|_{T}$. Define $\psi_{T}:\left.\mathcal{V} \rightarrow \mathcal{V}\right|_{T}$ to be the natural projection onto $\left.\mathcal{V}\right|_{T}$. Then, $\operatorname{dim} \Pi((A, T) ; v)=\#_{T}-\#_{A}$ for all $v$ in $\psi_{T}{ }^{-1}\left(F_{(A, T)}\right)$ which is obviously a generic

(12) Since the underlying preference is an expected utility function, $\Pi((A, T) ; v)$ is an affine subspace of $H(T)$. See Green and Osband (1991) for an extensive discussion of the structure of the state simplex in relation to the set of best responses when the underlying preference is expected utility representable. 
subset of $\mathcal{V}$. Define $=\cap_{\mathcal{c}}\left(\psi_{T}^{-1}\left(F_{(A, T)}\right)\right)$. Then, $F$ is clearly generic in $\mathcal{V}$ and property $\left.i\right)$ is proved.

The dimension of the affine hull of two affine subspaces $X$ and $Y$ is at most $\operatorname{dim} X+$ $\operatorname{dim} Y+1$. Applying this simple fact successively, the maximal possible dimension of $\Pi(\mathcal{C} ; v)$ is $\sum_{\mathcal{C}} \operatorname{dim} \Pi((A, T) ; v)+\#_{\mathcal{C}}-1$. For $v$ in $F$ as defined above, this number is $\sum_{\mathcal{C}}\left({ }^{\#} T-\#_{A}\right)+{ }^{\#}-1=\# \mathbf{T}-1-\chi(\mathcal{C} ; \mathbf{T})$. This proves property $\left.i i\right)$.

For certain utilities of the Receiver, $\pi \in \Pi(\mathcal{C} ; v)$ even if $\operatorname{dim} \Pi(\mathcal{C} ; v)<\# \mathbf{T}-1$ so that $\Pi(\mathcal{C} ; v)$ is a proper affine subspace of $H(\mathbf{T})$. One question we are concerned here is whether these utilities are only exceptional in the sense that they form a negligible set. Suppose to the contrary, that is, $\operatorname{dim} \Pi(\mathcal{C} ; v)<\# T-1$ and $\pi \in \Pi(\mathcal{C} ; v)$ for "almost all" utilities in a non-empty open subset of $\mathcal{V}$. Then, for each of these utilities, "almost all" neighboring priors do not belong to $\Pi(\mathcal{C} ; v)$, so that "almost all" neighboring priors do not have this property. In this sense, $\pi$ is special. However, this is contradictory to a finding due to next three Lemmas, namely, "all priors are equivalent in the sense that the set of utilities that satisfy (5.a) are identical for all priors up to certain rescaling of utility levels of the Receiver."

Given two priors $\pi$ and $\pi^{\prime}$ in $\Delta^{\circ}(\mathbf{T})$, define $f_{\left(\pi, \pi^{\prime}\right)}: \mathcal{V} \rightarrow \mathcal{V}$ as follows: Denoting $v^{\prime}=f_{\left(\pi, \pi^{\prime}\right)}(v)$

$$
v_{t}^{\prime a}=\left(\frac{\pi_{t}}{\pi_{t}^{\prime}}\right) v_{t}^{a}, \forall a \in \mathbf{A}, \forall t \in \mathbf{T}
$$

Lemma $5.3: \pi \in \Pi(\mathcal{C} ; v)$ if and only if $\pi^{\prime} \in \Pi\left(\mathcal{C} ; v^{\prime}\right)$ where $v^{\prime}=f_{\left(\pi, \pi^{\prime}\right)}(v)$.

Proof : Denote $\mathcal{C}=\left\{\left(A^{1}, T^{1}\right),\left(A^{2}, T^{2}\right), \ldots,\left(A^{K}, T^{K}\right)\right\}$. Suppose $\pi \in \Pi(\mathcal{C}, v)$. Since $\Pi\left(\left(A^{k}, T^{k}\right) ; v\right)$ is an affine subspace for each $\left(A^{k}, T^{k}\right) \in \mathcal{C}$, it means that $\pi=\sum_{k=1}^{K} \beta_{k} p^{k}$ where $\beta_{k} \in \mathbb{R}$ and $\sum_{k=1}^{K} \beta_{k}=1$, and $p^{k} \in \Pi\left(\left(A^{k}, T^{k}\right) ; v\right)$ for all $\left(A^{k}, T^{k}\right) \in \mathcal{C}$. We need to show analogous property for $\pi^{\prime}$ and $v^{\prime}$. Define $q^{k} \in H(\mathbf{T})$ by $q_{t}^{k}=\left(\frac{\pi_{t}^{\prime}}{\pi_{t}}\right) p_{t}^{k}$. Then, clearly $\pi^{\prime}=\sum_{k=1}^{K} \beta_{k} q^{k}$. It remains to show that $q^{k} \in \Pi\left(\left(A^{k}, T^{k}\right) ; v^{\prime}\right)$ for all $\left(A^{k}, T^{k}\right) \in \mathcal{C}$. Take $\left(A^{k}, T^{k}\right) \in \mathcal{C}$ and let $a, b \in A^{k}$. Then, $q^{k} \cdot v^{\prime a}=p^{k} \cdot v^{a}$ and $q^{k} \cdot v^{\prime b}=p^{k} \cdot v^{b}$. Since $p^{k} \in \Pi\left(\left(A^{k}, T^{k}\right) ; v\right)$, we have $p^{k} \cdot v^{a}=p^{k} \cdot v^{b}$. Therefore, $q^{k} \cdot v^{\prime a}=q^{k} \cdot v^{\prime b}$ so that $q^{k} \in \Pi\left(\left(A^{k}, T^{k}\right) ; v^{\prime}\right)$. This completes the proof that $\pi^{\prime} \in \Pi\left(\mathcal{C}, v^{\prime}\right)$ if $\pi \in \Pi(\mathcal{C}, v)$. 
Because of the exact symmetry between $\{\pi, v\}$ and $\left\{\pi^{\prime}, v^{\prime}\right\}$, above argument proves the other direction also by exchanging the roles of $\{\pi, v\}$ and $\left\{\pi^{\prime}, v^{\prime}\right\}$.

Clearly, $f_{\left(\pi, \pi^{\prime}\right)}$ is a homeomorphism whose inverse is $f_{\left(\pi^{\prime}, \pi\right)}$. In fact, it is a rescaling of the Receiver's utility level and the sets of utilities that satisfy (5.a) are identical for the two priors after this rescaling of utility levels. In particular, given $\pi$, if (5.a) is satisfied for an open set of utilities of the Receiver, then we can find a neighborhood of $\pi$ and an open set of utilities of the Receiver such that (5.a) is satisfied for any pair of prior and utility picked from these two sets. Lemma 5.5 formalizes this idea and Lemma 5.4 is a preliminary mathematical result.

Lemma 5.4 : Let $g: \Theta \times \mathbb{R}^{N} \rightarrow \mathbb{R}^{N}$ be a continuous function where $\Theta$ be an open subset of a Euclidean space. Suppose that, for all $\theta \in \Theta, g(\theta, \cdot): \mathbb{R}^{N} \rightarrow \mathbb{R}^{N}$ is a homeomorphism. Fix $\theta^{*}$ and a nonempty open subset $\mathcal{Z}^{0}$ of $\mathbb{R}^{N}$. Then, there exist an open neighborhood $\mathcal{O}$ of $\theta^{*}$ and a nonempty open subset $\mathcal{Z}^{1} \subset g\left(\theta^{*}, \mathcal{Z}^{0}\right)$ such that, for all $\theta \in \mathcal{O}, \mathcal{Z}^{1} \subset g\left(\theta, \mathcal{Z}^{0}\right)$.

Proof: Define $G: \Theta \times \mathbb{R}^{N} \rightarrow \Theta \times \mathbb{R}^{N}$ by $G(\theta, v)=(\theta, g(\theta, v))$. Then, $G$ is continuous, one to one and onto. Moreover, by Invariance of Domain, $G$ is an open map so that it is a homeomorphism.

Since $G\left(\Theta \times \mathcal{Z}^{0}\right)$ is open containing $\left(\theta^{*}, v^{*}\right)$ for some $v^{*} \in \mathcal{Z}^{0}$, we can find open sets $\mathcal{O} \subset \Theta$ and $\mathcal{Z}^{1} \subset \mathbb{R}^{N}$ such that $\mathcal{O} \times \mathcal{Z}^{1} \subset G\left(\Theta \times \mathcal{Z}^{0}\right)$. Take any $\theta \in \mathcal{O}$ and $v \in \mathcal{Z}^{1}$. Since $(\theta, v) \in G\left(\Theta \times \mathcal{Z}^{0}\right)$, there exists $v^{\prime} \in \mathcal{Z}^{0}$ such that $g\left(\theta, v^{\prime}\right)=v$.

Lemma 5.5: Let $\mathcal{C}$ be a configuration on $\{\mathbf{A}, \mathbf{T}\}$ and let $\pi^{*} \in \Delta(\mathbf{T})$. Suppose $\pi^{*} \in \Pi(\mathcal{C} ; v)$ for all $v \in \mathcal{V}^{0}$ where $\mathcal{V}^{0}$ is a nonempty open subset of $\mathcal{V}$. Then, there exists an open neighborhood $\mathcal{O}$ of $\pi^{*}$ and a nonempty open set $\mathcal{V}^{1} \subset \mathcal{V}^{0}$ such that

$$
\forall \pi \in \mathcal{O}, \forall v \in \mathcal{V}^{1}, \quad \pi \in \Pi(\mathcal{C} ; v)
$$

Proof: Define $g: \Delta^{\circ}(T) \times \mathcal{V} \rightarrow \mathcal{V}$ by

$$
g(\pi, v)=f_{\left(\pi^{*}, \pi\right)}(v)
$$


Clearly, $g(\pi, \cdot)$ is a homeomorphism. By Lemma 5.4, there exist an open neighborhood $\mathcal{O}$ of $\pi^{*}$ and a nonempty open subset $\mathcal{V}^{1} \subset g\left(\pi^{*}, \mathcal{V}^{0}\right)=\mathcal{V}^{0}$ such that, for all $\pi \in \mathcal{O}$, $\mathcal{V}^{1} \subset g\left(\pi, \mathcal{V}^{0}\right)$. By Lemma 5.3,

$$
\forall \pi \in \mathcal{O}, \forall v \in \mathcal{V}^{1}, \pi \in \Pi(\mathcal{C} ; v)
$$

Now, we are ready to prove Proposition 5.1.

Proof of Proposition 5.1: Let $\tilde{\mathcal{V}}$ be the set of utilities of the Receiver for which $\Pi(\mathcal{C} ; v)$ has a positive codimension in $H(\mathbf{T})$ and $\pi \in \Pi(\mathcal{C} ; v)$. Then, $\tilde{\mathcal{V}}$ is a semi-algebraic set by Remark 3.1. Suppose that $\tilde{\mathcal{V}}$ has an interior point, i.e., there is an open set $\mathcal{V}^{0}$ contained in $\tilde{\mathcal{V}}$. Then, by Lemma 5.5 , there is $\tilde{v} \in \mathcal{V}^{0} \subset \tilde{\mathcal{V}}$ such that $\Pi(\mathcal{C} ; \tilde{v})$ contains an open neighborhood of $\pi$, which is a contradiction to $\Pi(\mathcal{C} ; \tilde{v})$ having a positive codimension. Hence, $\tilde{\mathcal{V}}$ is a negligible set by Lemma 3.2. Therefore, there is a generic subset $G$ of $\mathcal{V}$ such that

$$
\text { for all } v \in G, \quad \pi \in \Pi(\mathcal{C} ; v) \text { only if } \Pi(\mathcal{C}, v)=H(\mathbf{T})
$$

If $\chi(\mathcal{C} ; \mathbf{T})>0, \operatorname{dim} \Pi(\mathcal{C}, v)<\# \mathbf{T}-1$ for generic $v$ by Lemma $5.2(i i)$. By (5.d), part (a) is proved.

Next, consider a configuration $\mathcal{C}$ which is trim with $\chi(\mathcal{C} ; \mathbf{T})=0$. Let $F$ be the generic subset of $\mathcal{V}$ in Lemma 5.2. Consider $v$ in $F \cap G$, a generic subset of $\mathcal{V}$, such that $\pi \in \Pi(\mathcal{C} ; v)$. Then, by $(5 . \mathrm{d}), \Pi(\mathcal{C}, v)=H(\mathrm{~T})$. By Lemma 5.2, this is possible only if the following holds for each $(A, T) \in \mathcal{C}: H\left(\cup_{(\mathcal{C} \backslash\{(A, T)\})} \Pi\left(\left(A^{\prime}, T^{\prime}\right) ; v\right)\right)$ and $\Pi((A, T) ; v)$ are disjoint affine subspace of dimensions \# $-1-\chi(\mathcal{C} \backslash\{(A, T)\} ; \mathbf{T})$ and ${ }^{\#} T-{ }_{A} A$, respectively, and the affine hull of them is $H(\mathbf{T})$. Therefore, for each $(A, T) \in \mathcal{C}$,

$$
H\left(H\left(\cup_{(\mathcal{C} \backslash\{(A, T)\})} \Pi\left(\left(A^{\prime}, T^{\prime}\right) ; v\right)\right) \cup\{\pi\}\right) \cap \Pi((A, T) ; v) \text { is a singleton }
$$

This means that the collection of intersection points in (5.e) of all bisupports in $\mathcal{C}$ is the only collection of posteriors that can be generated by a talking strategy satisfying (RO). Moreover, each posterior in this collection is independent of the rest of posteriors by (5.e) so that there is only one affine combination of these posteriors that produce $\pi$. Therefore, there is at most one talking strategy that satisfies (RO). In fact, there exists one only if all 
the posteriors are in $\Delta(\mathbf{T})$ and $\pi$ is expressed as a convex combination of these posteriors. This completes the proof.

We conclude by establishing the Criteria of Categorization stated in section 2 , thereby proving Theorem 2.2. In section 4, we established (C1) and (C2). Criterion (C3) is immedaite from Proposition 5.1 (a). Finally consider a just configuration $\mathcal{C}$ which is trim with $\chi(\mathcal{C} ; \mathbf{T})=0$. By Proposition $5.1(\mathrm{~b})$, there is at most one talking strategy satisfying (RO) for generic utilities of the Receiver. On the other hand, by Proposition 4.2 (a) or 4.8, for generic utilities of the Sender, there is at most one expectation vector, say $u^{*}$, that satisfies (SO). Moreover, for generic utilities, the induced utility plane of each bisupport is of full dimension so that there is a unique response that supports $u^{*}$ in the sense of (SO). Therefore, for generic utilities, the expectation vector (if there exists one) is implemented by a unique action strategy. Conclusively, given a just configuration $\mathcal{C}$ which is trim with $\chi(\mathcal{C} ; \mathbf{T})=0$, for generic utilities, at most one pair of talking and action strategies satisfies (SO) and (RO) with respect to $\mathcal{C}$. This establishes (C4). 


\section{REFERENCES}

Benedetti, R. and J.-J. Risler (1990), Real Algebraic and Semi Algebraic Sets, Hermann, Paris.

Blume, L.E. and W.R. Zame (1989), "The Algebraic Geometry of Perfect and Sequential Equilibrium," Cornell University Department of Economics, mimeo.

Cho, I.-K. and D. Kreps (1987), "Signalling Games and Stable Equilibria," Quarterly Journal of Economics, 102, 179-221.

Crawford, V. and J. Sobel (1982), "Strategic Information Transmission," Econometrica, 50, 1431-1452.

Debreu, G. (1970), "Economies with a Finite Set of Equilibria," Econometrica, 38, 387-392.

Farrell, J. (1985), "Credible Neologisms in Games of Communication," GTE Lab.

Green, E. J. and K. Osband (1991), "A Revealed Preference Theory for Expected Utility," Review of Economic Studies, 58, 677-696.

Green, J. and N. Stokey (1980), "A Two-Person Game of Information Transmission," Harvard University, Discussion Paper \#751.

Guillemin, V. and A. Pollack (1974), Differential Topology. Prentice-Hall, Inc., New Jersey. Harsanyi, J.C. (1973), "Oddness of the Number of Equilibrium Points: a New Proof," International Journal of Game Theory, 2, 235-250.

Kreps, D. and R. Wilson (1982), "Sequential Equilibria," Econometrica, 50, 863-894.

Matthews, S., M. Okuno-Fujiwara, and A. Postlewaite (1991), "Refining Cheap-Talk Equilibria," Journal of Economic Theory, 55, 247-273.

McLennan, A. (1991), "On the Generic Finiteness of Equilibrium Outcome Distributions in Game Forms," University of Minnesota Department of Economics, mimeo.

Schanuel, S.H., L.K. Simon, and W.R. Zame (1989), "The Algebraic Geometry of Games and the Tracing Procedure," SUNY at Buffalo, Mathematics Department, mimeo.

Whitney, H. (1954), "Elementary Structure of Real Algebraic Varieties," Annals of Mathematics, 66, 545-556. 